\title{
A systematic review of the effect of breakfast on the cognitive performance of children and adolescents
}

\author{
Alexa Hoyland, Louise Dye* and Clare L. Lawton \\ Human Appetite Research Unit, Institute of Psychological Sciences, University of Leeds, Leeds LS2 9JT, UK
}

Breakfast is recommended as part of a healthy diet because it is associated with healthier macroand micronutrient intakes, BMI and lifestyle. Breakfast is also widely promoted to improve cognitive function and academic performance, leading to the provision of breakfast initiatives by public health bodies. Despite this positive and intuitive perception of cognitive benefits, there has been no systematic review of the evidence. Systematic review methodology was employed to evaluate the effects of breakfast on cognitive performance in well-nourished children and nutritionally at-risk or stunted children. Acute experimental studies, school feeding programmes and studies of habitual breakfast intake are reviewed. Comparisons of breakfast $v$. no breakfast and breakfasts differing in energy and macronutrient composition are discussed. Included are forty-five studies described in forty-one papers published between 1950 and 2008. The evidence indicates that breakfast consumption is more beneficial than skipping breakfast, but this effect is more apparent in children whose nutritional status is compromised. There is a lack of research comparing breakfast type, precluding recommendations for the size and composition of an optimal breakfast for children's cognitive function. Few studies examined adolescents. Studies of school breakfast programmes suggest that such interventions can have positive effects on academic performance, but this may be in part explained by the increased school attendance that programmes encourage. The present systematic review considers methodological issues in this field and makes recommendations for future research design and policy priorities.

\section{Breakfast: Cognition: Children: Adolescents: Learning}

\section{Introduction}

Breakfast consumers tend to have higher micronutrient intakes, partly because of the fortification of breakfast cereals, and a better macronutrient profile than breakfast skippers $^{(1)}$. Regular breakfast cereal consumers have healthier body weights but also tend to engage in healthier lifestyle behaviours than those who skip breakfast ${ }^{(2)}$. Similarly, children who regularly eat breakfast tend to have a lower BMI and are less likely to be overweight than those who eat breakfast less frequently ${ }^{(3)}$. Studies in children suggest that breakfast eaters are more likely to meet daily nutrient intake guidelines compared with children who eat breakfast infrequently or skip breakfast ${ }^{(4)}$. Despite this, breakfast skipping increased in the USA from $14 \%$ to $25 \%$ between 1965 and $1991^{(5)}$. Moreover, the percentage of children eating breakfast in the UK has declined along with the nutrient quality of breakfast foods selected ${ }^{(6)}$, with implications for nutrient status and energy intake.

A good deal of research has considered the importance of breakfast consumption for cognitive performance ${ }^{(7)}$. Much of this research has been undertaken in healthy young adults, particularly undergraduate students. The premise that acute interventions can enhance mental performance in this population, in whom cognitive function is well protected, is now being reconsidered ${ }^{(8)}$. Increasingly, interest has turned towards groups who may be more vulnerable to nutritional deficits or cognitive impairment, for example, children and the elderly ${ }^{(9)}$.

Breakfast consumption, as with other meals, provides fuel for preferential oxidation of glucose. In children aged between 3 and 11 years, the brain has been shown to account for more than $50 \%$ of body oxygen consumption ${ }^{(10)}$. Children have a higher ratio of brain weight to liver weight (1.4-1.6 v. 0.73 in adults) and a $50 \%$ greater metabolic rate per unit brain weight. Thus children exert greater demands on glycogen stores during overnight fasts which are often longer than in adults. The child's relatively small muscle mass, in turn, limits the availability of glucogenic amino acids for hepatic gluconeogenesis ${ }^{(10)}$. Average global cerebral blood flow and $\mathrm{O}_{2}$ utilisation are 1.8 and 1.3 times higher in children than adults, respectively ${ }^{(11)}$. Positron emission tomography studies of thirty children, aged $0-18$ years, demonstrate that

Abbreviations: GI, glycaemic index; GL, glycaemic load.

* Corresponding author: Professor L. Dye, fax + 44113 3435749, email 1.dye@leeds.ac.uk 
the higher cerebral metabolic rate of glucose utilisation gradually declines from the age of 10 years, stabilising at age 16-18 years ${ }^{(12,13)}$. The higher metabolic turnover of children, their rapid growth rates and the importance of their cognitive function for academic achievement underlie the need for optimal nutrition.

Previous reviews give mixed support for the effects of breakfast provision in experimental and school settings on cognitive or scholastic performance ${ }^{(14-16)}$. Pollitt \& Mathews ${ }^{(17)}$ concluded that there was no convincing evidence for '. . either long or short term effects of breakfast on cognition and school learning...' (p. 804S). Common to these reviews is the inclusion of studies of variable scientific quality, the lack of a clear classification of effects across particular cognitive domains and the failure to identify confounds. These reviews have not systematically evaluated all available published studies selected on the basis of the quality of research design and do not acknowledge the very likely publication bias which results in publication of a greater proportion of studies with positive findings.

Two recent publications have systematically reviewed the effect of nutritional interventions, including school feeding programmes, on physical development, school attendance and performance. One considered a range of nutritional interventions, including breakfast, sugar intake on attention deficit-hyperactivity disorder, and fish oil supplementation $^{(18)}$, concluding that there was insufficient evidence to identify any effect of nutrition on learning of children from the developed world. In contrast, a Cochrane review of school meal provision to disadvantaged children suggested some small benefits for physical and psychosocial health ${ }^{(19)}$. Although the application of systematic review methodology to children's nutrition is timely and desirable ${ }^{(20)}$, there has not been a focused systematic review to evaluate the cognitive effects of breakfast in children. Such a review would inform government initiatives in the UK, the USA and elsewhere which aim to improve the diet of children with positive consequences for cognitive function.

The aim of the present review, therefore, was to provide a systematic examination of the best evidence from controlled studies of the effects of breakfast on the cognitive performance of school-aged children. The review examines whether the effects of breakfast on cognitive performance are consistent across populations of differing nutritional status with the aim of evaluating whether breakfast interventions can have an impact on the cognitive performance of well-nourished children in the developed world as well as those of or at risk of poor nutritional status. A further aim was to identify the nature of the breakfast which was associated with the clearest positive effects on cognitive function. Nutritional parameters which could account for breakfast effects on cognition include the macronutrient composition, energy provision and glycaemic properties of the breakfast meal.

\section{Literature search}

\section{Search strategy and search terms}

Electronic databases were searched on 20 January 2009. The databases queried were MedLine (1950 to January 2009),
PsycInfo (1967 to January 2009) and Web of Science (1955 to January 2009). Table 1 provides the search strings used as text words and keywords in each database. Additional search strategies involved scanning reference lists of review articles identified. This yielded three further articles. Following removal of duplicates ( $n$ 253), 350 citations were retrieved for possible inclusion in the present review.

\section{Inclusion and exclusion criteria}

Papers were included or excluded according to the following criteria.

Participants. Studies of children or adolescents (aged 4-18 years) of either sex were included. Studies were excluded if they examined adult, elderly or patient samples.

Manipulations. Any type of breakfast manipulation, including studies comparing breakfast with no breakfast, and studies of different breakfast types were included. Studies of the effects of glucose ingestion or of intake at other mealtimes were excluded. Breakfast was defined according to the descriptions of the meals or foods consumed provided in the papers reviewed. These varied but generally considered breakfast to be the first food consumed that day although this was not the case for some interventions where prior intake was not controlled. Studies were not excluded on the basis of the content of the meal; for example, studies that included interventions using drinks and/or snacks were included.

Outcome measures. Studies including any standardised outcome measures of cognitive performance were included. Studies solely examining fatigue or employing only qualitative measures of cognitive performance were excluded. Studies involving teachers' subjective ratings of

Table 1. Search terms

\section{Search string*}

Breakfast AND (children OR adolescents) AND cogniti\$

Breakfast AND (children OR adolescents) AND memory

Breakfast AND (children OR adolescents) AND attention

Breakfast AND (children OR adolescents) AND vigilance

Breakfast AND (children OR adolescents) AND reaction time

6 Breakfast AND (children OR adolescents) AND spatial

7 Breakfast AND (children OR adolescents) AND visuo-spatial

8 Breakfast AND (children OR adolescents) AND psychomotor

9 Breakfast AND (children OR adolescents) AND tracking

10 Breakfast AND (children OR adolescents) AND problem solving

Breakfast AND (children OR adolescents) AND logic

Breakfast AND (children OR adolescents) AND reasoning

Breakfast AND (children OR adolescents) AND recall

Carbohydrate AND (children OR adolescents) AND cogniti\$

Glucose AND (children OR adolescents) AND cogniti\$

Breakfast AND (children OR adolescents) AND cogniti\$ AND school

17 Breakfast AND (children OR adolescents) AND cogniti\$ AND program

*\$ denotes word truncation. 
performance were excluded since these do not provide an objective measure of cognitive function. Acute (where performance was assessed within $12 \mathrm{~h}$ of breakfast consumption) and habitual effects of breakfast manipulations (for example, school breakfast programmes) were included.

\section{Study selection process}

Figure 1 details the study selection process and the number of papers retrieved and excluded at each stage. Of the 350 studies retrieved, 300 exclusions were made, most commonly because the studies examined adult or elderly samples; assessed breakfast programme delivery or efficiency only; measured breakfast behaviours only; took measures of appetite only. Also excluded were nine review papers. Some papers contained multiple studies ${ }^{(21-24)}$. Therefore, forty-one articles were extracted providing fortyfive studies for review.

Each study appears in the data tables only once, irrespective of whether the data were reported in more than one paper (Grantham-McGregor ${ }^{(15)}$ was also reported in Chandler et al. ${ }^{(25)}$; Pollitt et al. ${ }^{(23)}$ Study 1 was also reported in Pollitt et al. ${ }^{(26)}$; Simeon \& GranthamMcGregor ${ }^{(27)}$ was also reported in Simeon \& GranthamMcGregor $^{(28)}$ and Simeon ${ }^{(29)}$ Study 2).

\section{Tabulation of studies}

Tables 2-5 were produced to summarise the main characteristics of each study. The studies were categorised according to the intervention duration (acute, long term or habitual) and the study participants (well nourished or of differing nutritional status). Representation by sex and age range as well as mean and SD and were included if details were provided. The study design was classified as repeated

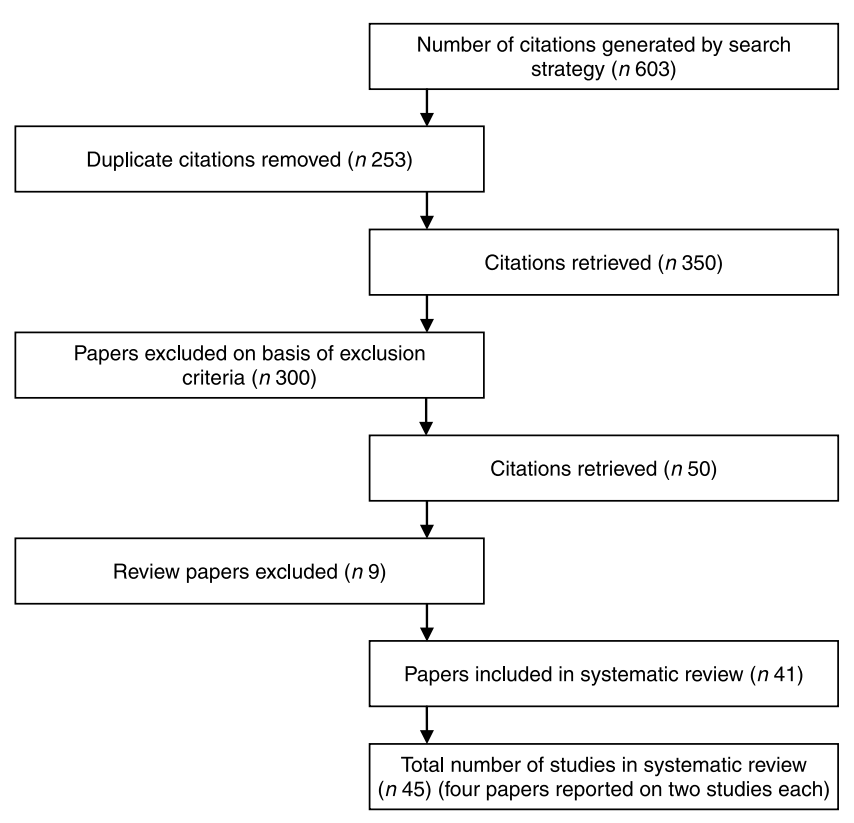

Fig. 1. Flow diagram of the study selection process. measures (where participants received each intervention) or independent groups (where participants were assigned to receive one intervention arm only). Randomisation, counterbalancing or cross-over strategies were noted if sufficient detail was evident to confirm that these experimental controls were employed. The duration of the test battery and the time post-breakfast consumption of administration were included in the tables if these could be determined. Explicitly stated fasting periods were recorded.

Cognitive tests were listed with their respective outcome measures (dependent variables) if specified. The cognitive domain assessed by each test is provided where description of the test features was sufficiently detailed.

Where enough detail was provided, the energy content of the breakfasts was calculated from the macronutrient composition of the interventions. The statistical analysis performed on the data by each study is also provided. The comments provided for each study in the tables consider study quality in terms of design, analysis and conclusions drawn and indicate the sponsor or funding body where provided.

\section{Quality assessment}

Each study was rated for quality using pre-defined assessment criteria by two of the authors independently. The inter-rater correlation for ratings was $r 0.85$ and discrepancies were discussed as a panel with the third author to reach consensus. Validated tools for the assessment of clinical trials (for example, Jadad et al. ${ }^{(30)}$ ) do not lend themselves to the design features and experimental manipulations or comparisons made by breakfast studies. Therefore, we devised an eighteen-item tool which covered key elements of study aims and design, sample selection, breakfast manipulation, controls, analysis and outcomes (see Appendix). This tool was based on others developed to assess barriers to healthy eating in children ${ }^{(31)}$ and parental decision making for child health ${ }^{(32)}$. All criteria were equally weighted and a score of 1 was obtained if the criterion was satisfied. Quality assessment (QA) ratings appear in Tables 2-5 next to each study reference. Due to insufficient details, five studies could not be assigned a QA rating (see Tables 2-5). We chose not to exclude studies on the basis of quality threshold because there was a limited number of studies in some categories and a large variation in the adequacy of descriptions provided. Hence we provide a quality assessment critique for each type of study.

\section{Results}

Studies are grouped into four categories according to the type of investigation undertaken. Of the forty-five studies, twentyeight were examinations of the acute effects of breakfast $v$. no breakfast or breakfast type on cognitive performance. This is broken down into studies in well-nourished children ( $n$ 21) and in children of differing nutritional status ( $n 7)$. Of the studies, thirteen examined the long-term effects of school breakfast programmes and breakfast clubs on cognitive performance. Lastly, four studies examined the effect of the quality of habitual breakfast intake on cognitive 


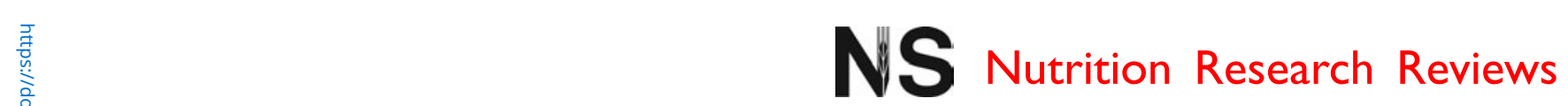

Table 2. Acute experimental intervention studies in well-nourished children (twenty-one studies, reported in nineteen papers)

\begin{tabular}{|c|c|c|c|c|c|c|c|}
\hline Reference & QA & Sample & Country & Design and intervention & Cognitive measures & Reported findings & Comments \\
\hline $\begin{array}{l}\text { Benton et al. } \\
\quad(2007)^{(41)}\end{array}$ & 11 & $\begin{array}{l}\text { Nineteen lower-SES school } \\
\text { children (nine male, ten } \\
\text { female), mean age } \\
6 \text { years } 10 \text { months, age } \\
\text { range } 5 \text { years } 11 \text { months } \\
\text { to } 7 \text { years } 8 \text { months }\end{array}$ & UK & $\begin{array}{l}\text { 4-week school BF club. } \\
\text { Unbalanced RM with } \\
\text { three breakfasts } \\
\text { designed to be } \\
\text { equi-energetic } \\
\text { (a) High GL: cornflakes, } \\
\text { semi-skimmed milk, } \\
\text { sugar, waffle, maple } \\
\text { syrup. Mean } 820 \text { (SD } \\
\text { 335) kJ } \\
\text { (b) Medium GL: } \\
\text { scrambled egg, bread, } \\
\text { jam, low-fat spread, } \\
\text { low-energy yogurt. } \\
\text { Mean } 703 \text { (SD 159) kJ } \\
\text { (c) Low GL: ham, cheese, } \\
\text { soya and linseed } \\
\text { bread, low-fat spread. } \\
\text { Mean } 657 \text { (SD } 439 \text { ) kJ } \\
\text { Amount offered more } \\
\text { than consumed } \\
\text { BF at } 08.15 \text { until } 08.45 \\
\text { hours, blind testing at } \\
10.35 \text { until } 11.45 \text { hours }\end{array}$ & $\begin{array}{l}\text { Memory (I\&D): } \\
\text { British Ability Scale } \\
\text { object recall (verbal) } \\
\text { Object location recall } \\
\text { (spatial) } \\
\text { Sustained attention: } \\
\text { Shakow paradigm: } \\
\text { respond to light } \\
\text { stimulus with auditory } \\
\text { warning } 3 \mathrm{~s} \text { or } 12 \mathrm{~s} \\
\text { prior }\end{array}$ & $\begin{array}{l}\text { ANOVA: no effect on } \\
\text { memory or attention } \\
\text { Correlations: GL } \\
\text { inversely related to } \\
\text { immediate (not } \\
\text { delayed) memory. } \\
\text { GL had effect on } \\
\text { attention under } \\
\text { specific conditions } \\
\text { (improved on } 2 \text { nd } \\
\text { test with } 12 \mathrm{~s} \\
\text { warning) }\end{array}$ & $\begin{array}{l}\text { Breakfasts differed sig- } \\
\text { nificantly in macronu- } \\
\text { trient content. Large } \\
\text { intake variability } \\
\text { Design unbalanced. } \\
\text { Independence } \\
\text { assumption not met, } \\
\text { degrees of freedom } \\
\text { do not concur } \\
\text { with design } \\
\text { High risk of type } 1 \text { error in } \\
\text { multiple correlational } \\
\text { analyses } \\
\text { BBC }\end{array}$ \\
\hline $\begin{array}{l}\text { Busch et al. } \\
\qquad(2002)^{(34)}\end{array}$ & 16 & $\begin{array}{l}\text { Twenty-one boys aged 9- } \\
12 \text { years, normal weight. } \\
\text { No: medication, learning } \\
\text { disability, dietary restric- } \\
\text { tions }\end{array}$ & USA & $\begin{array}{l}\text { Counterbalanced cross- } \\
\text { over with two BF con- } \\
\text { ditions: } \\
\text { (a) } 25 \mathrm{~g} \text { confectionery } \\
\text { snack (mainly simple } \\
\text { CHO) } \\
\text { (b) Half cup aspartame } \\
\text { drink matched for } \\
\text { sweetness } \\
\text { 1-week washout } \\
45 \text { min CT battery } \\
\text { at }+15 \text { min post-BF }\end{array}$ & $\begin{array}{l}\text { Attention: } \\
\text { CPT } \\
\text { Memory: } \\
\text { Map task (spatial) } \\
\text { Story recall (verbal) } \\
\text { Digit span (working) } \\
\text { Visual perception: } \\
\text { Rey Complex Figure } \\
\text { Copy }\end{array}$ & $\begin{array}{l}\text { Attention: better after } \\
\text { snack than drink. } \\
\text { Snack decreased } \\
\text { number of false } \\
\text { alarms and } \\
\text { prevented increase in } \\
\text { misses with time on } \\
\text { task } \\
\text { No effects on other } \\
\text { tests }\end{array}$ & $\begin{array}{l}\text { Monetary incentive } \\
\text { to parents } \\
\text { Mars Inc. }\end{array}$ \\
\hline $\begin{array}{l}\text { Connors \& } \\
\text { Blouin } \\
(1983)^{(14)}\end{array}$ & 8 & $\begin{array}{l}\text { Ten children aged } 9-11 \\
\text { years }\end{array}$ & USA & $\begin{array}{l}\text { RM with BF (milk, cereal } \\
\text { with sugar, egg, juice } \\
\text { and toast) and no-BF } \\
\text { conditions } \\
\text { Each condition adminis- } \\
\text { tered twice after } 12 \mathrm{~h} \\
\text { fast } \\
30 \text { min CT battery at } \\
09.50,11.00 \text { and } \\
12.10 \text { hours }\end{array}$ & $\begin{array}{l}\text { CPT } \\
\text { Arithmetic test } \\
\text { EEG visual evoked } \\
\quad \text { potentials test }\end{array}$ & $\begin{array}{l}\text { CPT: fewer errors and } \\
\text { less variability } \\
\text { after BF } \\
\text { Arithmetic: better } \\
\text { performance } \\
\text { mid-morning after BF } \\
\text { EEG: BF reduced } \\
\text { cardiac acceleration } \\
\text { and amplitude of } \\
\text { evoked potentials }\end{array}$ & $\begin{array}{l}\text { EEG effects suggest BF } \\
\text { may influence neural } \\
\text { processes mediating } \\
\text { performance }\end{array}$ \\
\hline $\begin{array}{l}\text { Cromer et al. } \\
(1990)^{(37)}\end{array}$ & 17 & $\begin{array}{l}\text { Thirty-four middle-class, } \\
\text { suburban high school } \\
\text { children, overall mean } \\
\text { age } 14.2 \text { (SD 0.4) years }\end{array}$ & USA & $I G$ & Memory: & $\begin{array}{l}\text { No effect of BF } \\
\text { condition on } \\
\text { cognition }\end{array}$ & $\begin{array}{l}\text { Monetary incentive } \\
\text { to participants }\end{array}$ \\
\hline
\end{tabular}




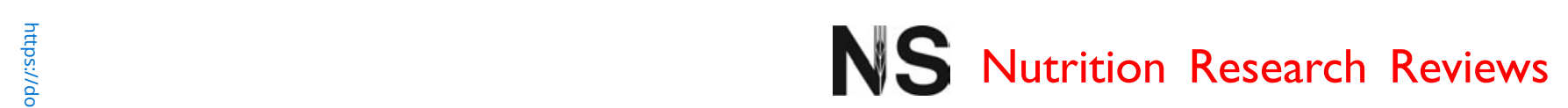

Table 2. Continued

\begin{tabular}{|c|c|c|c|c|c|c|c|}
\hline Reference & QA & Sample & Country & Design and intervention & Cognitive measures & Reported findings & Comments \\
\hline $\begin{array}{c}\text { Morris \& Sarll } \\
(2001)^{(69)}\end{array}$ & 14 & $\begin{array}{l}\text { Eighty A level students } \\
\text { (thirty-six female, forty- } \\
\text { four male), mean age } \\
21 \cdot 2 \text { (SD } 4.4 \text { ) years, mini- } \\
\text { mum age } 17 \text { years }\end{array}$ & UK & $\begin{array}{l}\text { IG, blind, random } \\
\text { allocation to: } \\
\text { (a) } 300 \mathrm{ml} \text { orange juice } \\
\text { ( } 50 \mathrm{~g} \text { glucose in } 250 \mathrm{ml} \\
\text { water, } 40 \mathrm{ml} \text { orange } \\
\text { squash }+10 \mathrm{ml} \text { lemon } \\
\text { juice), or } \\
\text { (b) } 300 \mathrm{ml} \text { orange juice } \\
\text { (2 } \mathrm{g} \text { Sweetex in } 250 \mathrm{ml} \\
\text { water, } 40 \mathrm{ml} \text { orange } \\
\text { squash }+10 \mathrm{ml} \text { lemon } \\
\text { juice) } \\
\text { Matched drinks } \\
\text { CT immediately before } \\
\text { and }+20 \text { min after } \\
\text { drink }\end{array}$ & $\begin{array}{l}\text { Daneman-Carpenter } \\
\text { listening span test } \\
\text { (immediate recall) }\end{array}$ & $\begin{array}{l}\text { Interaction of drink } \times \\
\text { time. Recall } \\
\text { improved } \\
\text { significantly after } \\
\text { glucose drink }\end{array}$ & \\
\hline $\begin{array}{l}\text { Pollitt et al. } \\
\qquad(1981)^{(35)}\end{array}$ & 16 & $\begin{array}{l}\text { Thirty-four children (twenty- } \\
\text { two female, twelve } \\
\text { male), mean age } 10 \\
\text { years } 4 \text { months, age } \\
\text { range } 9-11 \text { years, } \\
\text { between } 10 \text { and } 90 \text { per- } \\
\text { centile for weight, mostly } \\
\text { prepubertal, medical } \\
\text { diagnosis of 'good } \\
\text { health' }\end{array}$ & USA & $\begin{array}{l}\text { RM counterbalanced in- } \\
\text { patient stay with BF } \\
\text { and no-BF conditions } \\
\text { BF: waffles and syrup, } \\
\text { margarine, orange } \\
\text { juice, milk ( } 2238 \mathrm{~kJ} \text {, } \\
15 \mathrm{~g} \text { protein, } 20 \mathrm{~g} \mathrm{fat,} \\
75 \mathrm{~g} \mathrm{CHO}) \\
\text { Standard evening meal } \\
\text { at } 17.00 \text { hours, blood } \\
\text { sample at } 21.00 \text { and } \\
12.00 \text { hours } \\
\text { CT testing at }+180 \text { min } \\
\text { Peabody Picture } \\
\text { Vocabulary task used } \\
\text { to measure IQ }\end{array}$ & $\begin{array}{l}\text { Visual perception: } \\
\text { MFFT } \\
\text { Attention: } \\
\text { CPT - HCl task } \\
\text { (sequential memory } \\
\text { for objects) }\end{array}$ & $\begin{array}{l}\text { MFFT: benefit of BF } \\
\text { in lower IQ only. } \\
\text { Decrease in glucose } \\
\text { associated with more } \\
\text { errors } \\
\text { HCl: fewer errors after } \\
\text { no BF but only for } \\
\text { those whose blood } \\
\text { glucose was lower in } \\
\text { no-BF than in BF } \\
\text { condition }\end{array}$ & $\begin{array}{l}\text { Large energy } \\
\text { intervention yet few } \\
\text { effects on } \\
\text { performance } \\
\text { IQ median split used as } \\
\text { factor in analysis } \\
\text { Ford Foundation }\end{array}$ \\
\hline $\begin{array}{l}\text { Pollitt et al. } \\
(1982-3)^{(38)}\end{array}$ & 14 & $\begin{array}{l}\text { Thirty-nine children (twenty } \\
\text { female, nineteen male), } \\
\text { mean age } 10 \text { years } \\
4 \text { months, age range } \\
9-11 \text { years, between } \\
10 \text { and } 90 \text { percentile } \\
\text { for weight, mostly } \\
\text { prepubertal, } \\
\text { medical diagnosis of } \\
\text { 'good health' }\end{array}$ & USA & $\begin{array}{l}\text { RM counterbalanced } \\
\text { in-patient stay with BF } \\
\text { and no-BF conditions } \\
\text { BF: waffles and syrup, } \\
\text { margarine, orange } \\
\text { juice, milk }(1874 \mathrm{~kJ}, \\
12 \mathrm{~g} \text { protein, } 16 \mathrm{~g} \text { fat, } \\
65 \mathrm{~g} \mathrm{CHO}) \\
\text { Standard evening meal } \\
\text { at } 17.00 \text { hours, BF at } \\
08.00 \text { hours } \\
\text { Blind CT testing } \\
\text { at }+180 \text { min } \\
\text { Peabody Picture Voca- } \\
\text { bulary task used to } \\
\text { measure IQ }\end{array}$ & $\begin{array}{l}\text { Visual perception: } \\
\text { MFFT } \\
\text { Attention: } \\
\text { HCl } \\
\text { Short-term memory: } \\
\text { Xylophone tapping } \\
\text { Digit span }\end{array}$ & $\begin{array}{l}\text { MFFT: more errors after } \\
\text { no BF than BF on } \\
\text { hard version only } \\
\text { HCl: better recall after } \\
\text { no BF than BF but IQ } \\
\text { not included in } \\
\text { analysis }\end{array}$ & 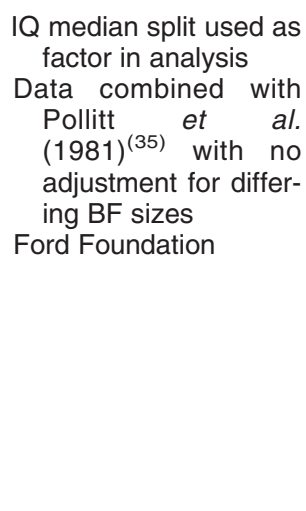 \\
\hline
\end{tabular}




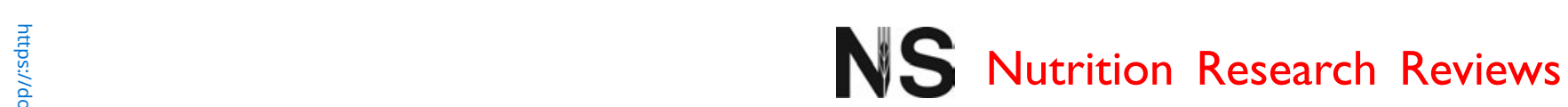

Table 2. Continued

\begin{tabular}{|c|c|c|c|c|}
\hline Reference & QA & Sample & Country & Design and intervention \\
\hline $\begin{array}{l}\text { Pollitt et al. } \\
\text { (1998) }\end{array}$ & 14 & $\begin{array}{l}\text { Thirty-two children (twenty- } \\
\text { three female, nine male), } \\
\text { aged } 9-11 \text { years, } \\
\text { between } 10 \text { and } 90 \% \\
\text { percentile for weight, } \\
\text { mostly prepubertal, } \\
\text { medical diagnosis of } \\
\text { 'good health' }\end{array}$ & USA & $\begin{array}{l}\text { RM counterbalanced } \\
\text { in-patient stay with BF } \\
\text { ( } 2238 \mathrm{~kJ}, 15 \mathrm{~g} \text { protein, } \\
20 \mathrm{~g} \text { fat, } 75 \mathrm{~g} \mathrm{CHO}) \\
\text { and no-BF conditions } \\
\text { Standard evening meal } \\
\text { at } 17.00 \text { hours, BF at } \\
08.00 \text { hours } \\
\text { Blind CT testing at } \\
+180 \text { min } \\
\text { Peabody Picture } \\
\text { Vocabulary task used } \\
\text { to measure IQ }\end{array}$ \\
\hline $\begin{array}{c}\text { Vaisman etal. } \\
\qquad(1996)^{(36)}\end{array}$ & 14 & $\begin{array}{l}569 \text { children (279 female, } \\
290 \text { male) from different } \\
\text { SES, age range } 11-13 \\
\text { years }\end{array}$ & Israel & $\begin{array}{l}\text { Unbalanced IG } \\
\text { Baseline: BF at home or } \\
\text { no BF (fasted) } \\
\text { 14 d chronic intervention: } \\
\text { BF at school } \\
\text { (two-thirds) or no BF } \\
\text { instruction (one-third) } \\
\text { BF: } 30 \mathrm{~g} \text { sugared } \\
\text { cornflakes }+200 \mathrm{ml} \\
\text { ( } 3 \% \text { fat) milk } \\
\text { CT testing }+120 \text { min } \\
\text { post-BF (BF at home) } \\
\text { or }+30 \text { min post-BF } \\
\text { (BF at school) }\end{array}$ \\
\hline
\end{tabular}

\begin{tabular}{ll} 
Cognitive measures & Reported findings \\
\hline Visual perception: & MFFT: poorer \\
MFFT & performance after no \\
Attention: & BF than BF in lower- \\
HCl & IQ group only \\
CPT & HCl: better recall in no \\
& BF than BF \\
& CPT: no effect
\end{tabular}

Comments

IQ median split used as

factor in analysis

$(2238 \mathrm{~kJ}, 15 \mathrm{~g}$ protein,

Cl: better recall in

CPT: no effect

08.00 hours

Blind CT testing at
$+180 \mathrm{~min}$

eabody Pictu

Vocabulary task used

to measure IQ

Baseline: BF at home or

Memory:

RAVLT (I\&D,

Benton Visual Reten-

Benton Visual Reten-
tion test (visuospatial)

Wechsler Memory

Scale revised - story

(logical)

(BF at school)

$\begin{array}{cc}\begin{array}{c}\text { Wesnes et al. } \\ (2003)^{(33)}\end{array} & 14 \quad \begin{array}{c}\text { Twenty-nine children of } \\ \text { similar SES; fifteen }\end{array} \\ & \text { female, mean age } 12 \cdot 3 \\ & \text { years, age range } 9-16 \\ & \text { years; fourteen male, } \\ & \text { mean age } 12 \cdot 1 \text { years, } \\ & \text { age range } 9-16 \text { years }\end{array}$

UK mean age 12.1 years age range $9-16$ years
RM with four conditions over four consecutive days:

(a) Shreddies

$(45 \mathrm{~g})+$ semi-

skimmed milk $(125 \mathrm{ml})$ $854 \mathrm{~kJ}, 38.3 \mathrm{~g} \mathrm{CHO}$ ( $25.2 \mathrm{~g}$ complex $\mathrm{CHO}$ $6.9 \mathrm{~g}$ sucrose, $6.25 \mathrm{~g}$ lactose)

(b) Cheerios $(30 \mathrm{~g})+$ semi-skimmed milk $(125 \mathrm{ml}): 686 \mathrm{~kJ}, 28.7 \mathrm{~g}$ CHO, $6.4 \mathrm{~g}$ sucrose $6.25 \mathrm{~g}$ lactose)
Word recall (I\&D)

SRT

Digit vigilance

Spatial memory

Numeric memory

Word recognition

Picture recognition
Baseline: better immediate verbal recall after BF. No differences on acquisition, delayed recognition and delayed recall factors

Post-intervention:

RVALT: better mean learning, best learning, retroactive inhibition and recognition $\mathrm{BF}$ at home and no
B BF at home and no $\mathrm{BF}$. Better delayed
recall and temporal order for BF at school than BF at home only Benton and Wechsler: better after BF at school than BF at home No differences on factors

General pattern of performance decline after no BF across morning. Cereal breakfasts

attenuated decline

Factor-based analysis: cereal breakfasts better for power of attention and quality factors. Trends for other factors
Unbalanced design. Weighted means used to account fo the unequal cell sizes. Dropout from each condition not reported

No consideration of

$$
\text { SES }
$$

Secondary analysis: principal components to derive factors reflecting

performance. Crosssectional analysis difficult to separate from post-intervention analysis

Scores on previously derived factors used as outcomes

No analysis of individual tests provided

No differences between the cereal breakfast Age not included as a covariate in analysis Monetary incentive to participants

Cereal Partners, UK 


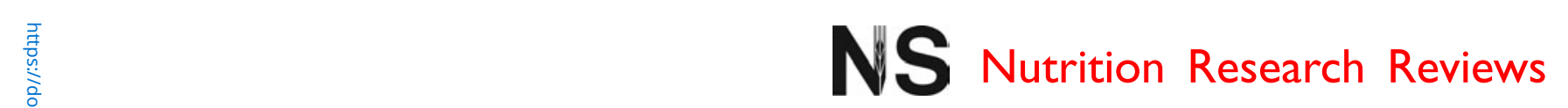

Table 2. Continued

\begin{tabular}{|c|c|c|c|c|c|c|c|}
\hline Reference & QA & Sample & Country & Design and intervention & Cognitive measures & Reported findings & Comments \\
\hline & & & & $\begin{array}{l}\text { (c) Orange-flavoured } \\
\text { drink ( } 330 \mathrm{ml}): 602 \mathrm{~kJ} \text {, } \\
38 \cdot 3 \mathrm{~g} \mathrm{CHO} \text { (glucose) } \\
\text { (d) No BF } \\
25 \mathrm{~min} \mathrm{CT} \text { testing at }+20 \text {, } \\
+80,+140,+200 \mathrm{~min} \\
\text { post-BF }\end{array}$ & & $\begin{array}{l}\text { Immediate word recall } \\
\text { at } 12.00 \text { hours: } \\
\text { No BF: }-12 \% \text {; glucose: } \\
-27 \% ; \\
\text { Cheerios: }+3 \% ; \\
\text { Shreddies: }+5 \% \\
\text { (relative to baseline) }\end{array}$ & \\
\hline $\begin{array}{l}\text { Widenhorn- } \\
\text { Müller etal. } \\
(2008)^{(70)}\end{array}$ & 15 & $\begin{array}{l}104 \text { children (fifty female, } \\
\text { fifty-four male), aged } \\
17 \cdot 2 \text { (SD 1.6) years, } \\
\text { range } 13-20 \text { years; } \\
88 \% \text { regular BF eaters } \\
\text { (i.e. five or more times } \\
\text { per week) }\end{array}$ & Germany & $\begin{array}{l}\text { RM counterbalanced } \\
\text { cross-over with } 7 \mathrm{~d} \\
\text { wash-out } \\
\text { (a) BF: } 60 \mathrm{~g} \text { wholewheat } \\
\text { bread, } 20 \mathrm{~g} \text { butter, } 20 \mathrm{~g} \\
\text { nougat spread, } 30 \mathrm{~g} \\
\text { strawberry jam, } \\
\text { ad libitum } \\
\text { water and unswee- } \\
\text { tened peppermint tea } \\
\text { (1992 kJ) } \\
\text { (b) No BF } \\
\text { BF at } 07.30 \text { hours. CT } \\
\text { at }+45 \text { min post-BF } \\
\text { Unbalanced design with } \\
\text { respect to treatment } \\
\text { order: } 91 \text { BF-no BF; } \\
67 \text { no BF-BF }\end{array}$ & $\begin{array}{l}\text { Attention: } \\
\text { d2 test } \\
\text { (visual search) } \\
\text { Memory: } \\
\text { Object recall } \\
\text { Trail route (spatial) } \\
\text { Logos (picture recall) } \\
\text { Turkish vocabulary } \\
\text { (recognition) } \\
\text { Telephone numbers } \\
\text { (paired associate) } \\
\text { Fact cued recall }\end{array}$ & $\begin{array}{l}\text { d2: trend for improved } \\
\text { concentration after } \\
\text { BF but BF } \times \text { order } \\
\text { interaction } \\
\text { Memory: positive effect } \\
\text { of BF in males for trail } \\
\text { task but presence of } \\
\text { order effects and no } \\
\text { significant effects on } \\
\text { subtests } \\
\text { No effects in females } \\
\text { except trend for posi- } \\
\text { tive effect of BF in } \\
\text { telephone number } \\
\text { task with presence } \\
\text { of order effect }\end{array}$ & $\begin{array}{l}\text { For d2 task } n 70 \\
\text { Separate analysis by } \\
\text { sex } \\
\text { Order of testing and } \\
\text { unbalanced design } \\
\text { make evaluation of } \\
\text { effects of BF on mul- } \\
\text { tiple tests included } \\
\text { difficult }\end{array}$ \\
\hline $\begin{array}{l}\text { Wyon et al. } \\
(1997)^{(71)}\end{array}$ & 8 & $\begin{array}{l}195 \text { suburban school chil- } \\
\text { dren aged } 10 \text { years }\end{array}$ & $\begin{array}{l}\text { Sweden } \\
\text { and } \\
\text { Denmark }\end{array}$ & $\begin{array}{l}\text { IG with 'good' and 'bad' } \\
\text { BF conditions eaten at } \\
\text { home } \\
\text { (a) Good: high variety, } \\
\text { good macronutrient } \\
\text { balance. Selected } \\
\text { from yoghurt/sour milk } \\
\text { product, cereal, sand- } \\
\text { wiches with cheese or } \\
\text { liver paste, orange } \\
\text { juice, milk, tea or hot } \\
\text { chocolate. Mean } \\
2130 \text { kJ } \\
\text { (b) Bad: lacking in variety, } \\
\text { low protein. Typically a } \\
\text { sweet drink, bread, } \\
\text { jam or sweetbread. } \\
\text { Mean } 840 \text { kJ } \\
\text { CT in late morning }\end{array}$ & $\begin{array}{l}\text { Addition } \\
\text { Multiplication } \\
\text { Grammatical logic } \\
\text { Number checking } \\
\quad \text { (visual search) } \\
\text { Reading - speed and } \\
\text { comprehension } \\
\text { Word recognition } \\
\text { Creativity }\end{array}$ & $\begin{array}{l}\text { Better speed and fewer } \\
\text { errors on creativity } \\
\text { test after the 'good' } \\
\text { BF }\end{array}$ & $\begin{array}{l}\text { Various reports of these } \\
\text { data with differing } \\
\text { sample sizes and } \\
\text { findings available } \\
\text { Swedish National Dairy } \\
\text { Association }\end{array}$ \\
\hline
\end{tabular}

QA, quality assessment; SES, socio-economic status; BF, breakfast; RM, repeated measures; GL, glycaemic load; I\&D, immediate and delayed (memory); BBC, British Broadcasting Corporation; CHO, carbohydrate; CT, cognitive test(s); CPT, Continuous Performance Test; EEG, electro-encephalography; IG, independent groups; MFFT, Matched Familiar Figures Test; MAST, Memory and Search Test; GI, glycaemic index; $\mathrm{SRT}$, simple reaction time; CRT, choice reaction time; np, quality assessment not possible; RTE, ready-to-eat; SBP, school breakfast programme; IQ, intelligence quotient; HCI, Hagen Central Incidental Task; RAVLT,

Rey Auditory-Verbal Learning Test. 


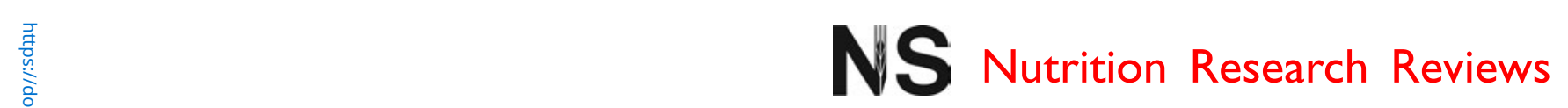

Table 3. Acute experimental intervention studies in children with differing nutritional status (seven studies)

\begin{tabular}{|c|c|c|c|c|c|c|c|}
\hline Reference & QA & Sample & Country & Design and intervention & Cognitive measure & Reported findings & Comment \\
\hline $\begin{array}{l}\text { Cueto et al. } \\
(1998)^{(72)}\end{array}$ & 16 & $\begin{array}{l}\text { Fifty-four male subjects } \\
\text { classified as: } \\
\text { (1) At risk: }<-1 \mathrm{SD} \\
\text { NCHS height for age } \\
\text { and }<-0.5 \mathrm{SD} \\
\text { weight for height }(n 23) \\
\text { (2) Not at risk }(n 31)\end{array}$ & Peru & $\begin{array}{l}\text { RM counterbalanced } \\
\text { cross-over in-patient } \\
\text { stay with two } \\
\text { conditions: } \\
\text { (a) BF: } 80 \mathrm{~g} \text { cake }+50 \mathrm{~g} \\
\text { protein drink }(2134 \mathrm{~kJ} \text {; } \\
82 \mathrm{~g} \mathrm{CHO}, 14 \mathrm{~g} \\
\text { protein, } 12 \mathrm{~g} \mathrm{fat} \text {, } \\
7.2 \mathrm{mg} \mathrm{Fe}, 240 \mu \mathrm{g} \\
\text { vitamin A, } 27 \mathrm{mg} \\
\text { vitamin C) } \\
\text { (b) No BF } \\
\text { Standard evening meal } \\
\text { ( } 2510 \mathrm{~kJ}) \\
\text { BF at } 08.00 \text { hours } \\
\text { CT at } 11.00 \text { hours. Blood } \\
\text { glucose } \\
\text { measured during } \\
\text { testing }\end{array}$ & $\begin{array}{l}\text { Number discrimination } \\
\text { (visual search) } \\
\text { Sternberg memory } \\
\text { search (short-term } \\
\text { memory) } \\
\text { Peabody Picture Voca- } \\
\text { bulary Test (long- } \\
\text { term memory and } \\
\text { verbal ability) } \\
\text { Raven's Progressive } \\
\text { Matrices } \\
\text { CRT } \\
\text { Stimulus discrimination }\end{array}$ & $\begin{array}{l}\text { Stimulus discrimination } \\
\text { and Sternberg: } \\
\text { slower decision time } \\
\text { after no BF in at-risk } \\
\text { group only }\end{array}$ & $\begin{array}{l}\text { Order entered as } \\
\text { covariate in analysis } \\
\text { Participants received } \\
\text { gifts for participating } \\
\text { Kellogg's Co. and } \\
\text { Government of Peru }\end{array}$ \\
\hline $\begin{array}{l}\text { Grantham- } \\
\text { McGregor } \\
\text { et al. } \\
(1998)^{(73)} \\
\text { and } \\
\text { Chandler } \\
\text { et al. } \\
(1995)^{(74)}\end{array}$ & 9 & $\begin{array}{l}197 \text { subjects classified as: } \\
\text { (1) Undernourished } \\
(-1 \text { sD below NCHS } \\
\text { weight-for-age stan- } \\
\text { dard) ( } n \text { 97) } \\
\text { (2) Well-nourished } \\
(n 100) \\
\text { Groups matched on } \\
\text { school, class and sex }\end{array}$ & Jamaica & $\begin{array}{l}\text { RM counterbalanced } \\
\text { cross-over with two } \\
\text { conditions: } \\
\text { (a) BF (at school, no } \\
\text { details given) } \\
\text { (b) Control (slice } \\
\text { of orange) } \\
\text { CT } 2 \text { weeks apart, } \\
\text { shortly after BF }\end{array}$ & $\begin{array}{l}\text { Visual search } \\
\text { Digit span } \\
\text { Categoric fluency } \\
\text { Speed of decision } \\
\text { making }\end{array}$ & $\begin{array}{l}\text { Fluency: better after BF } \\
\text { but only in undernour- } \\
\text { ished group } \\
\text { No effects on other tests }\end{array}$ & $\begin{array}{l}\text { No details of } \\
\text { composition or } \\
\text { energy content of BF } \\
\text { Nestlé Nutrition Support } \\
\text { Grant }\end{array}$ \\
\hline $\begin{array}{l}\text { López et al. } \\
(1993)^{(42)}\end{array}$ & 11 & $\begin{array}{l}279 \text { low-SES children } \\
\text { (134 male, } 145 \\
\text { female), mean age } \\
10 \text { years } 3 \text { months } \\
\text { (SD } 0.5 \text { years); classi- } \\
\text { fied as normal ( } n 106), \\
\text { wasted ( } n 73) \text { or } \\
\text { stunted }(n 100)\end{array}$ & Chile & $\begin{array}{l}\text { IG with two conditions: } \\
\text { (a) BF: two cakes, } \\
200 \mathrm{ml} \text { flavoured milk } \\
\text { (1648 kJ, } 6 \text { g protein) } \\
\text { (b) No BF } \\
24 \text { min CT at }+60 \mathrm{~min} \\
\text { post-BF }\end{array}$ & $\begin{array}{l}\text { Digit span } \\
\text { Domino test (problem } \\
\text { solving) } \\
\text { Attention test }\end{array}$ & $\begin{array}{l}\text { No effect of treatment } \\
\text { Stunted children made } \\
\text { more errors than nor- } \\
\text { mal or wasted children } \\
\text { on attention test }\end{array}$ & $\begin{array}{l}\text { ANCOVA performed } \\
\text { with IQ, SES, food } \\
\text { intake on previous } \\
\text { day and glycaemia } \\
\text { level as covariates } \\
\text { Nestlé Nutrition and } \\
\text { Ministry of } \\
\text { Education, Chile }\end{array}$ \\
\hline $\begin{array}{l}\text { Muthayya } \\
\text { et al. } \\
(2007)^{(75)}\end{array}$ & 16 & $\begin{array}{l}\text { Seventy-three children } \\
\text { of different SES: } \\
\text { (1) Thirty-four low SES } \\
\text { (nineteen female, } \\
\text { fifteen male), mean } \\
\text { age } 7.6 \text { (SD 0.6) } \\
\text { years. } 32 \% \text { wasted, } \\
21 \% \text { stunted } \\
\text { (2) Thirty-five high SES } \\
\text { (thirteen female, } \\
\text { twenty-two male), } \\
\text { mean age } 7.6 \\
\text { (SD 1.1) years }\end{array}$ & India & $\begin{array}{l}\text { RM counterbalanced, } \\
\text { three equi-energetic } \\
\text { conditions with } \\
\text { 1-week washout } \\
\text { (3515 kJ total): } \\
\text { (A) Small BF ( } 782 \mathrm{~kJ}) \text {, } \\
\text { snack ( } 640 \mathrm{~kJ}), \text { stan- } \\
\text { dard lunch ( } 2092 \mathrm{~kJ}) \\
\text { (B) Standard BF } \\
\text { (1423 kJ), snack, } \\
\text { small lunch ( } 1452 \mathrm{~kJ}) \\
\text { (C) Control: standard } \\
\text { BF, no snack, stan- } \\
\text { dard lunch } \\
\text { BF: chapatti and potato } \\
\text { curry }\end{array}$ & $\begin{array}{l}\text { Memory: } \\
\text { Picture recognition } \\
\text { (I\&D) } \\
\text { Attention: } \\
\text { CPT } \\
\text { Psychomotor: } \\
\text { Finger tapping }\end{array}$ & $\begin{array}{l}\text { Low SES: better immedi- } \\
\text { ate recognition after } \\
\text { condition B than } \\
\text { control at }+150 \text { min. } \\
\text { Condition B slowed } \\
\text { performance decline. } \\
\text { Decline in delayed } \\
\text { recognition attenuated } \\
\text { by snack in condition } \\
\text { A compared with } \\
\text { control. Fewer false } \\
\text { alarms after snack in } \\
\text { condition B compared } \\
\text { with control } \\
\text { High SES: greater decline } \\
\text { in immediate }\end{array}$ & $\begin{array}{l}\text { Separate analyses } \\
\text { for SES groups. } \\
\text { Low SES more } \\
\text { vulnerable in memory } \\
\text { domain only. Energy } \\
\text { distribution important - } \\
\text { snack at mid-morning } \\
\text { attenuated decline } \\
\text { Low SES given more } \\
\text { energy than usual } \\
\text { Unilever NL }\end{array}$ \\
\hline
\end{tabular}




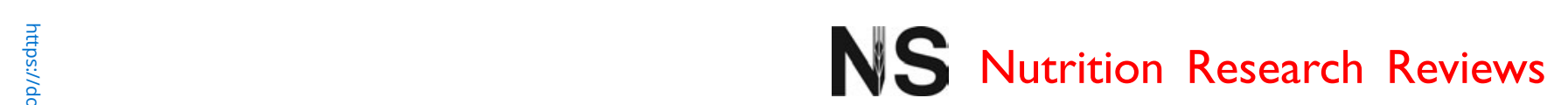

Table 3. Continued

\begin{tabular}{|c|c|c|c|c|c|c|c|}
\hline Reference & QA & Sample & Country & Design and intervention & Cognitive measure & Reported findings & Comment \\
\hline & & & & $\begin{array}{l}\text { Snack: mango-flavoured } \\
\text { co-extruded bar } \\
\text { Lunch: vegetable rice, } \\
\text { chickpea curry and } \\
\text { vermicelli dessert } \\
\text { BF at } 08.00 \text { hours, snack } \\
\text { at } 10.30 \text { hours } \\
22 \text { min CT at baseline, } \\
+30 \mathrm{~min},+150 \text { min, } \\
+300 \mathrm{~min}\end{array}$ & & $\begin{array}{l}\text { recognition but fewer } \\
\text { false alarms } \\
\text { after condition B than } \\
\text { control } \\
\text { Fewer false alarms after } \\
\text { snack in condition A or } \\
\text { B compared with control } \\
\text { No effects for CPT or } \\
\text { tapping. }\end{array}$ & \\
\hline $\begin{array}{l}\text { Noriega } \\
\qquad(2000)^{(76)}\end{array}$ & $\mathrm{np}$ & $\begin{array}{l}900 \text { children; } 450 \text { rural, } \\
450 \text { urban (extreme } \\
\text { poverty) }\end{array}$ & South America & $\begin{array}{l}\text { Four IG (BF = SBP): } \\
\text { (1) BF rural }(n 300) \\
\text { (2) No BF rural }(n 150) \\
\text { (3) BF urban }(n 300) \\
\text { (4) No BF urban }(n 150)\end{array}$ & $\begin{array}{l}\text { Test of attention and } \\
\text { discrimination mem- } \\
\text { ory }\end{array}$ & $\begin{array}{l}\text { Faster time and better } \\
\text { performance after BF } \\
\text { for rural only. No effect } \\
\text { in urban children }\end{array}$ & Detail lacking \\
\hline $\begin{array}{l}\text { Pollitt et al. } \\
\text { (1996) })^{(23)} \\
\text { and Pollitt } \\
\text { et al. } \\
(1998)^{(26)}\end{array}$ & 12 & $\begin{array}{l}\text { Fifty-four children (aged } \\
9-11 \text { years): } \\
\text { (1) At risk ( }-1 \text { SD height } \\
\text { for age and }-0.5 \text { SD } \\
\text { weight for height) } \\
(n 23) \\
\text { (2) Not at risk ( } n \text { 31) }\end{array}$ & Peru & $\begin{array}{l}\text { RM counterbalanced in- } \\
\text { patient stay with two } \\
\text { conditions: } \\
\text { (a) BF: } 80 \mathrm{~g} \text { cake }+50 \mathrm{~g} \\
\text { Amilac (similar to milk) } \\
\text { (b) No BF: diet soda } \\
\text { without caffeine } \\
\text { Standard evening meal } \\
\text { at } 17.00 \text { hours, BF at } \\
08.00 \text { hours } \\
\text { Blind CT at }+180 \text { min } \\
\text { Peabody Picture Voca- } \\
\text { bulary task used to } \\
\text { measure IQ }\end{array}$ & $\begin{array}{l}\text { Number discrimination } \\
\text { Raven Progressive } \\
\text { Matrices } \\
\text { RT } \\
\text { Stimulus discrimination } \\
\text { Sternberg Memory } \\
\text { Search }\end{array}$ & $\begin{array}{l}\text { Slower short-term mem- } \\
\text { ory in Sternberg and } \\
\text { discrimination tasks } \\
\text { after no BF in at-risk } \\
\text { children only. No other } \\
\text { effects }\end{array}$ & $\begin{array}{l}\text { No analysis strategy } \\
\text { stated }\end{array}$ \\
\hline $\begin{array}{l}\text { Simeon et al. } \\
(1989)^{(27)} \text {, } \\
\text { Simeon \& } \\
\text { Grantham- } \\
\text { McGregor } \\
(1987)^{(28)} \\
\text { and } \\
\text { Simeon } \\
(1998) \\
\text { Study } 2^{(29)}\end{array}$ & 15 & $\begin{array}{l}\text { Ninety children (age } \\
\text { range 9-10.5 years) } \\
\text { in three groups: } \\
\text { (1) Thirty malnourished } \\
\text { children (nineteen } \\
\text { male, eleven female), } \\
\text { hospitalised for mal- } \\
\text { nutrition in } 1 \text { st } 2 \text { years } \\
\text { (2) Thirty stunted chil- } \\
\text { dren (fifteen male, fif- } \\
\text { teen female) (height } \\
\text { for age }-2 \text { sD) } \\
\text { (3) Control group of thirty } \\
\text { non-stunted children } \\
\text { (fifteen male, fifteen } \\
\text { female) }\end{array}$ & Jamaica & $\begin{array}{l}\text { RM counterbalanced, } \\
\text { cross-over in-patient } \\
\text { stay with: } \\
\text { (a) BF: Nutribun, milk } \\
\text { and cheese }(2469 \mathrm{~kJ}, \\
91 \mathrm{~g} \mathrm{CHO}, 29 \mathrm{~g} \text { pro- } \\
\text { tein, } 12 \mathrm{~g} \text { fat) } \\
\text { (b) No BF: } 185 \mathrm{ml} \text { tea, } \\
\text { aspartame swee- } \\
\text { tened } \\
\text { Standard evening meal } \\
\text { at } 17.00 \text { hours, BF at } \\
08.00 \text { hours } \\
45 \text { min blind CT at } \\
+180 \text { min } \\
\text { Peabody Picture Voca- } \\
\text { bulary task used to } \\
\text { measure IQ }\end{array}$ & $\begin{array}{l}\text { Wechsler IQ scale for } \\
\text { children: } \\
\text { Arithmetic } \\
\text { Digit span } \\
\text { Coding (short-term } \\
\text { memory) } \\
\text { Fluency and listening } \\
\text { comprehension } \\
\text { (attention, auditory } \\
\text { short-term memory, } \\
\text { comprehension) } \\
\text { MFFT } \\
\text { HCI }\end{array}$ & $\begin{array}{l}\text { Fluency, coding, digit } \\
\text { span (backwards), } \\
\text { MFFT: poorer after no } \\
\text { BF in malnourished } \\
\text { groups than controls } \\
\text { Arithmetic: better after no } \\
\text { BF in controls only }\end{array}$ & $\begin{array}{l}\text { Only control and } \\
\text { stunted groups } \\
\text { matched for sex and } \\
\text { residence area } \\
\text { Nestlé Nutrition } \\
\text { Research Grant, The } \\
\text { Wellcome Trust, } \\
\text { Grace Kennedy \& } \\
\text { Co. and Restaurants } \\
\text { of Jamaica Ltd }\end{array}$ \\
\hline
\end{tabular}

QA, quality assessment; NCHS, National Center for Health Statistics; RM, repeated measures; BF, breakfast; CHO, carbohydrate; CT, cognitive test(s); CRT, choice reaction time; IG, independent groups; ANCOVA, analysis of covariance; SES, socio-economic status; IQ, intelligence quotient; I\&D, immediate and delayed (memory); CPT, Continuous Performance Test; np, quality assessment not possible; SBP, school breakfast programme; RT, reaction time; MFFT, Matched Familiar Figures Test; HCl, Hagen Central Incidental Task. 
performance. Of the forty-five studies, only ten examined participants over the age of 13 years.

\section{Studies of acute effects of breakfast in well-nourished children}

There were twenty-one studies of the acute effects of breakfast in well-nourished children identified and included in the review (see Table 2). Of the studies, nine were performed in the USA and six were from the UK. Of these studies, four came from the same two research groups. The rest were conducted in Europe, Israel, China and Venezuela. Details provided by each article varied considerably.

A repeated-measures design was employed by thirteen studies. A large number of cognitive measures were employed. Some cognitive domains were examined more frequently; for example, six studies employed continuous performance tests which assess sustained attention, and nine studies used other measures of attention such as visual search and checking tasks. Of the studies, nine employed tests of verbal memory and seven employed spatial memory. Digit span was used in six studies. Not all studies assessed more than one aspect of cognitive function and the timing of tests post-ingestion also varied. Studies often considered more than two treatments, commonly including breakfast and no-breakfast conditions within the design. The inclusion of multiple breakfast conditions within the same (often small) sample has implications for the statistical analysis such that degrees of freedom and power are reduced.

Effects of breakfast $\mathrm{v}$. no breakfast. The majority of these studies demonstrate positive effects of breakfast compared with no breakfast. However, effects vary over cognitive domain. Benefits of breakfast consumption were most evident on measures of memory and in terms of fewer errors on attention tasks especially later in the morning when performance decrements become apparent on the nobreakfast conditions. Effects on memory and attention are also clearest because more studies have used the same or comparable measures across these domains.

When verbal memory was assessed following breakfastno-breakfast interventions, six studies report null findings and four studies show positive effects of breakfast. Of the six studies which assessed spatial memory, three report a benefit of breakfast consumption, two show better performance in the no-breakfast condition and one shows no difference. In the study by Wesnes et al. ${ }^{(33)}$, it is not possible to distinguish verbal and non-verbal memory performance because a composite factor 'quality of episodic memory' (derived by principal components analysis) is reported. On this factor, there is a notable advantage for breakfast over no breakfast but immediate or delayed verbal performance cannot be distinguished from other memory measures. However, factors were derived from studies in adults and factor scores were not weighted for loading on the factors. Hence the factor structure and loadings may not be the same in children.

There are three well-designed studies that showed no effects of breakfast $v$. no breakfast comparisons on verbal memory ${ }^{(22,34,35)}$. Each used a repeated-measures design and the breakfasts provided between $753 \mathrm{~kJ}^{(22)}$ and $2238 \mathrm{~kJ}^{(35)}$.
Mahoney et al. ${ }^{(22)}$ demonstrated positive effects of breakfast using a spatial memory task but not with story recall (verbal memory). Vaisman et al. ${ }^{(36)}$ showed positive effects of breakfast at school compared with breakfast at home or no breakfast on immediate verbal recall. However, this study was an unbalanced design with no consideration of change over time or time of testing post-consumption, which differed for breakfast consumed at school or home.

Wesnes et al. ${ }^{(33)}$ also documented an effect of breakfast v. no breakfast on his 'quality of episodic memory' factor. Age was not included as a covariate in the analysis although the study included children aged from 9 to 16 years. The matching familiar figures test was not sensitive in Cromer et al.'s ${ }^{(37)}$ study of children with average IQ but more errors were made on the harder version of the task ${ }^{(38)}$ and in children with lower $\mathrm{IQ}^{(17)}$ after no breakfast. Visual perception might only be susceptible to nutritional intervention in more vulnerable samples.

Therefore, breakfast $v$. no breakfast comparisons show some positive benefits particularly if testing occurs later in the morning, with the effect more easily discernible where tests are more demanding and consider error rates.

Comparisons of different breakfasts. Fewer studies find effects on cognitive function when different breakfasts are compared. In total, nine studies compared at least two breakfasts that provided solid food, for example, cereals, doughnuts or toast. However, published studies do not always provide sufficiently detailed descriptions of the breakfasts that were administered to permit calculation of exact energy and macronutrient composition. Two studies ${ }^{(22,33)}$ compared cereal breakfasts with similar energy contents but which varied in terms of carbohydrate quality. Wesnes et al. ${ }^{(33)}$ included cereals that varied in amount of complex carbohydrate and Mahoney et al. ${ }^{(2)}$ compared an oatmeal breakfast against a ready-to-eat cereal. The two breakfast comparisons in each study were advantageous relative to the no-breakfast and glucose-drink conditions but performance did not differ between the cereal breakfasts in either study.

Only one study has explicitly compared the glycaemic index (GI) of two breakfasts ${ }^{(39)}$, but details of GI determination were not provided. GI may be calculated or estimated from international tables of GI values ${ }^{(40)}$, but potential differences in the glycaemic responses of adults and children are not well understood since GI studies have not been conducted in children. Glycaemic load (GL) of breakfasts was considered in one study of 6- to 7-year-old children $^{(41)}$. GL is calculated by multiplying the amount of available carbohydrate in a food item by the GI of the food and dividing this by 100 . The three breakfasts provided by Benton et al. ${ }^{(41)}$ varied in GL from 2.5 to 17.86 but more importantly the energy content of the breakfasts prescribed also varied from 657 to $820 \mathrm{~kJ}$ and actual intake was not consistent between participants.

Time of testing post-consumption may be important. The strongest effects have been found in the late morning, at $+130 \min ^{(39)}$ and $+200 \min ^{(33)}$. Mahoney et al. ${ }^{(22)}$ tested only at $1 \mathrm{~h}$ post-consumption and found an effect of breakfast $v$. no breakfast but no strong effects between two types of breakfast. Pollitt et al. ${ }^{(17,35)}$ administered one 
WS Nutrition Research Reviews

Table 4. School breakfast programmes (SBP) in well-nourished children and children of differing nutritional status (thirteen studies)

\begin{tabular}{|c|c|c|}
\hline Reference & QA & Sample \\
\hline $\begin{array}{l}\text { Cueto \& } \\
\text { Chinen } \\
(2008)^{(46)}\end{array}$ & 14 & $\begin{array}{l}590 \text { children (4th grade } \\
\text { 'full-' and ' } \\
\text { multi-grade' schools } \\
\text { with two-thirds }-2 \\
\text { SD NCHS height for } \\
\text { age), two groups: } \\
\text { (1) SBP, } 300 \text { children } \\
\text { (52\% male, } 48 \% \\
\text { female), mean age } \\
11.87 \text { (SD 1.77) years } \\
\text { (2) No intervention, } 290 \\
\text { children (50\% male, } \\
50 \% \text { female), mean } \\
\text { age } 11.87 \text { (SD 1.9) } \\
\text { years }\end{array}$ \\
\hline $\begin{array}{l}\text { Kleinman } \\
\text { et al. } \\
(2002)^{(77)}\end{array}$ & 13 & $\begin{array}{l}\text { Ninety-seven 3rd-6th } \\
\text { grade children of low } \\
\text { SES with } 70 \% \text { eligible } \\
\text { for free meals, } \\
\text { two groups: } \\
\text { (1) Low nutrient intake } \\
(n 29) \\
\text { (2) Not at risk }(n 68)\end{array}$ \\
\hline $\begin{array}{l}\text { Lieberman } \\
\text { et al. } \\
\quad(1976)^{(78)}\end{array}$ & 11 & $\begin{array}{l}617 \text { children, 3rd-6th } \\
\text { grade, predominantly } \\
\text { black, low income, } \\
\text { well nourished }\end{array}$ \\
\hline $\begin{array}{l}\text { Meyers et al. } \\
(1989)^{(79)}\end{array}$ & 13 & $\begin{array}{l}1023 \text { children, 3rd-6th } \\
\text { grade, predominantly } \\
\text { Hispanic, two groups: } \\
\text { (1) SBP, } 335 \text { children; } \\
163 \text { female, } 171 \text { male } \\
\text { (2) Non-SBP, } 688 \\
\text { children; } 340 \text { female, } \\
347 \text { male }\end{array}$ \\
\hline $\begin{array}{l}\text { Murphy et al. } \\
(1998)^{(80)}\end{array}$ & 15 & $\begin{array}{l}133 \text { children, 3rd-8th } \\
\text { grades, predomi- } \\
\text { nantly black, low } \\
\text { income; three groups } \\
\text { of SBP participation: } \\
\text { (1) Rarely (eighty-three } \\
\text { children; thirty-six } \\
\text { male, forty-seven f } \\
\text { emale } \\
\text { (2) Sometimes (twenty- } \\
\text { six children; twelve } \\
\text { male, fourteen } \\
\text { female) }\end{array}$ \\
\hline
\end{tabular}

Country and interign

G with two conditions:

(a) SBP at school after earlier BF at home. Cup of milk-like drink (no lactose), six smal biscuits $(2510 \mathrm{~kJ}$; $22.5 \mathrm{~g}$ protein, $20 \mathrm{~g}$ fat) provided $60 \%$ of RDA for various vitamins and minerals, $100 \%$ RDA Fe

(b) No intervention - BF at home only

BF at $10.00-11.00$ hours CT on $1 \mathrm{~d}$ of ongoing SBP

IG with CT pre- and post- 6 month SBP intervention

BF: no details

IG 8 months SBP intervention:

(a) School BF

IG with two conditions (SBP or non-SBP)

3-month intervention with CT pre and post BF: no details

IG with three conditions (rarely, sometimes or often frequency of SBP participation) or SBP participation) or non-SBP)

CT pre- and post-intervention

BF: no details

\begin{tabular}{lc}
\multicolumn{1}{c}{ Cognitive } & \multicolumn{1}{c}{$\begin{array}{c}\text { Reported } \\
\text { measures }\end{array}$} \\
findings
\end{tabular}

Comment

Analysis controlled for bilingualism/Spanish proficiency

Both groups likely to eat $\mathrm{BF}$ at home before walking to school

Lower SES in no-SBP group

Better school attendance in SBP group

Effects only in multigrade pupils

Data on academic performance from $n 79$ only

Project Bread/The Walk for Hunger, Boston, USA

Baseline scores were from school year before study

William T Grant Foundation, NY and Helmut Wolfgang Schumann Foundation,

Hannover, USA

Only objective performance measure was mathematics grade 


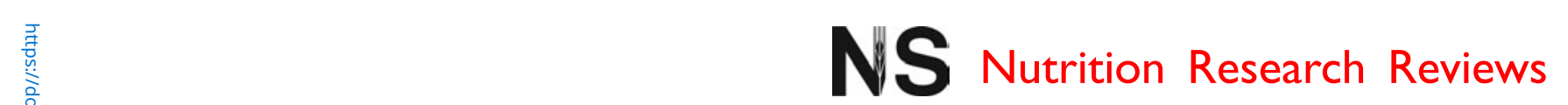

Table 4. Continued

\begin{tabular}{|c|c|c|c|c|c|c|c|}
\hline Reference & QA & Sample & Country & $\begin{array}{l}\text { Design } \\
\text { and intervention }\end{array}$ & $\begin{array}{l}\text { Cognitive } \\
\text { measures }\end{array}$ & $\begin{array}{l}\text { Reported } \\
\text { findings }\end{array}$ & Comment \\
\hline & & $\begin{array}{l}\text { (3) Often (twenty-four } \\
\text { children; ten male, } \\
\text { fourteen female) }\end{array}$ & & & & & \\
\hline $\begin{array}{l}\text { Pollitt et al. } \\
(1996)^{(23)}\end{array}$ & 7 & $\begin{array}{l}\text { Sixty children, 4th and } \\
\text { 5th grade recipients } \\
\text { of Government lunch } \\
\text { programme to be } \\
\text { provided BF }\end{array}$ & $\begin{array}{l}\text { Peru } \\
\quad \text { (Huaraz, } \\
3300 \mathrm{~m} \\
\text { elevation) }\end{array}$ & $\begin{array}{l}\text { IG with two conditions: } \\
\text { (a) 1-month SBP inter- } \\
\text { vention (increased } \\
\text { energy, protein and Fe } \\
\text { intake) } \\
\text { (b) No SBP }\end{array}$ & $\begin{array}{l}\text { Digit discrimination } \\
\text { Reading } \\
\text { comprehension } \\
\text { Vocabulary } \\
\text { Mathematics } \\
\text { Wechsler IQ scale: } \\
\text { Coding } \\
\text { Digit span }\end{array}$ & $\begin{array}{l}\text { Positive effect across } \\
\text { time on vocabulary } \\
\text { but no significant } \\
\text { effect of SBP alone }\end{array}$ & $\begin{array}{l}\text { Attendance increased in } \\
\text { experimental group } \\
\text { and decreased in } \\
\text { controls } \\
\text { No analysis strategy } \\
\text { stated } \\
\text { Kellogg's, USA }\end{array}$ \\
\hline $\begin{array}{l}\text { Richter et al. } \\
\left(_{(1997)^{(43)}}\right.\end{array}$ & 12 & $\begin{array}{l}108 \text { children (aged 5-7 } \\
\text { years) in two groups: } \\
\text { (1) SBP group: } \\
\text { undernourished farm } \\
\text { school (socially } \\
\text { disadvantaged), } \\
\text { fifty-five children, } \\
\text { aged } 10.5 \text { (SD } 1.9 \text { ) } \\
\text { years, age range } \\
7-14 \text { years } \\
\text { (2) Controls, well-nour- } \\
\text { ished inner city } \\
\text { school, fifty-five } \\
\text { children aged } 8.3 \\
\text { (sD } 0.8 \text { ) years, age } \\
\text { range } 7-10 \text { years }\end{array}$ & South Africa & $\begin{array}{l}\text { IG 6-week intervention } \\
\text { with pre- and post-test- } \\
\text { ing and two conditions: } \\
\text { (a) SBP group } \\
\text { (b) Non-SBP group } \\
\text { BF: no details given }\end{array}$ & $\begin{array}{l}\text { Attention } \\
\text { Distractibility } \\
\text { Short-term memory }\end{array}$ & $\begin{array}{l}\text { Increase in perform- } \\
\text { ance from pre- to } \\
\text { post-intervention in } \\
\text { SBP group }\end{array}$ & $\begin{array}{l}\text { No detail of subtests } \\
\text { Unmatched groups. } \\
\text { Experimental group } \\
\text { were significantly } \\
\text { older and more } \\
\text { undernourished than } \\
\text { controls, and had a } \\
\text { different background. } \\
\text { Not a fair comparison }\end{array}$ \\
\hline $\begin{array}{l}\text { Shemilt et al. } \\
(2004)^{(45)}\end{array}$ & 12 & $\begin{array}{l}8209 \text { children (predomi- } \\
\text { nantly white) in two } \\
\text { groups: } \\
\text { (1) SBP intervention: } \\
69 \% \text { free school } \\
\text { meal entitlement, } \\
\text { mean age } 9.6 \text { (SD } \\
2.96 \text { ) years } \\
\text { (2) Controls, } 64 \% \text { free } \\
\text { school meal } \\
\text { entitlement, mean } \\
\text { age } 10.13 \text { (SD 3.9) } \\
\text { years }\end{array}$ & UK & $\begin{array}{l}\text { IG with two conditions: } \\
\text { (a) 3-month funding for } \\
\text { school BF club } \\
\text { (b) No funding }\end{array}$ & Reitan trail-making test & $\begin{array}{l}\text { Time to complete trail- } \\
\text { making part A was } \\
\text { faster in school BF } \\
\text { group at } 3 \text { months } \\
\text { Reduced absenteeism } \\
\text { in school BF group }\end{array}$ & $\begin{array}{l}\text { Intention-to-treat analysis } \\
\text { with group, baseline, } \\
\text { sex and free meal } \\
\text { eligibility as explana- } \\
\text { tory variables in } \\
\text { adjusted analysis } \\
\text { Contamination between } \\
\text { study arms } \\
\text { DoH, UK }\end{array}$ \\
\hline $\begin{array}{l}\text { Simeon } \\
\quad(1998)^{(29)}\end{array}$ & 9 & $\begin{array}{l}115 \text { children aged } \\
12-13 \text { years, poor } \\
\text { rural school }\end{array}$ & Jamaica & $\begin{array}{l}\text { IG with three conditions: } \\
\text { (a) School BF: cake and } \\
100 \mathrm{ml} \text { milk }(1602 \mathrm{~kJ}) \text { or } \\
\text { meat pie and } 100 \mathrm{ml} \\
\text { milk ( } 3059 \mathrm{~kJ}), n 44 \\
\text { (b) Syrup drink: } 134 \mathrm{~kJ}, n 33 \\
\text { (c) No BF: } n 38 \\
\text { BF at } 09.00 \text { hours } \\
\text { CT at start of } 1 \mathrm{st} \text { semester, }\end{array}$ & $\begin{array}{l}\text { Wide-range achieve- } \\
\text { ment test including } \\
\text { arithmetic, spelling } \\
\text { and reading }\end{array}$ & $\begin{array}{l}\text { No difference between } \\
\text { syrup drink and no } \\
\text { BF, so combined in } \\
\text { analysis } \\
\text { Increased attendance } \\
\text { in school BF group } \\
\text { Higher arithmetic } \\
\text { scores in school } \\
\text { BF group. Effect }\end{array}$ & $\begin{array}{l}\text { Commonwealth } \\
\text { Caribbean Research } \\
\text { Council }\end{array}$ \\
\hline
\end{tabular}




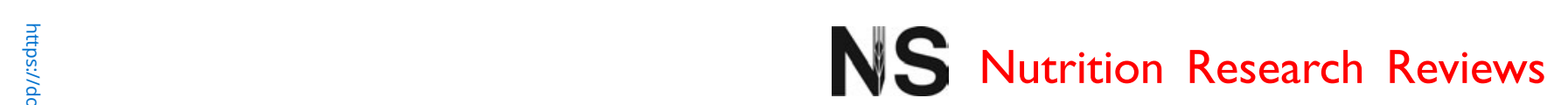

Table 4. Continued

\begin{tabular}{|c|c|c|c|c|c|c|c|}
\hline Reference & QA & Sample & Country & $\begin{array}{c}\text { Design } \\
\text { and intervention }\end{array}$ & $\begin{array}{l}\text { Cognitive } \\
\text { measures }\end{array}$ & $\begin{array}{l}\text { Reported } \\
\text { findings }\end{array}$ & Comment \\
\hline & & & & $\begin{array}{l}\text { start and end of 2nd } \\
\text { semester }\end{array}$ & & $\begin{array}{l}\text { maintained when } \\
\text { attendance and } \\
\text { weight gain } \\
\text { controlled for }\end{array}$ & \\
\hline $\begin{array}{l}\text { Vera Noriega } \\
\text { et al. } \\
(2000)^{(44)}\end{array}$ & $\mathrm{np}$ & $\begin{array}{l}450 \text { children, aged } 4-6 \\
\text { years, extremely } \\
\text { poor areas }\end{array}$ & Mexico & $\begin{array}{l}\text { IG with pre- and } \\
\text { post-testing. Two } \\
\text { conditions: } \\
\text { (a) School BF: no } \\
\text { details given, } n 300 \\
\text { (b) No school BF, } n 150 \\
\text { No details of intervention } \\
\text { duration }\end{array}$ & $\begin{array}{l}\text { Tests of memory, atten- } \\
\text { tion and cognition }\end{array}$ & $\begin{array}{l}\text { No-school-BF group } \\
\text { had better scores at } \\
\text { baseline. Post-test } \\
\text { performance similar } \\
\text { in both groups } \\
\text { School BF group } \\
\text { showed improved } \\
\text { stimulus selection } \\
\text { and reproduction } \\
\text { (memory) from pre to } \\
\text { post. No effect on RT }\end{array}$ & $\begin{array}{l}\text { Insufficient detail about } \\
\text { tests }\end{array}$ \\
\hline $\begin{array}{l}\text { Wahlstrom \& } \\
\text { Begalle } \\
{(1999)^{(81)}}^{(19)}\end{array}$ & 4 & $\begin{array}{l}2901 \text { children of } \\
\text { elementary school } \\
\text { age }\end{array}$ & USA & $\begin{array}{l}\text { 3-year intervention BF } \\
\text { programme } v \text {. no BF } \\
\text { programme }\end{array}$ & $\begin{array}{l}\text { Standardised achieve- } \\
\text { ment tests }\end{array}$ & $\begin{array}{l}\text { School BF associated } \\
\text { with general increase } \\
\text { in mathematics and } \\
\text { reading scores }\end{array}$ & $\begin{array}{l}\text { Reports selective find- } \\
\text { ings only }\end{array}$ \\
\hline 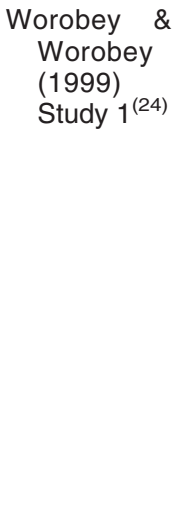 & 12 & $\begin{array}{l}\text { Twelve children (five } \\
\text { female, seven male), } \\
\text { age range } 3 \text { years } 10 \\
\text { months to } 5 \text { years } 2 \\
\text { months, middle class }\end{array}$ & USA & $\begin{array}{l}\text { 2-week baseline } \\
\text { assessment with } \\
\text { random allocation to CT } \\
\text { in week } 1 \text { or } 2 \text {. } \\
\text { Followed by } 6 \text {-week } \\
\text { school BF intervention } \\
\text { Compared baseline (BF at } \\
\text { home) with post-inter- } \\
\text { vention (BF at school) } \\
\text { School BF: similar in } \\
\text { energy to usual BF at } \\
\text { home (1096 kJ and } \\
1151 \mathrm{~kJ}) \text {. Higher in } \\
\text { CHO, lower in sugar } \\
20-30 \text { min CT } \\
\text { at }+30 \text { min after BF }\end{array}$ & $\begin{array}{l}\text { Mazes (psychomotor) } \\
\text { Embedded figures } \\
\text { (restructuring ability) } \\
\text { Pattern match } \\
\text { Same or different } \\
\text { (visual perception } \\
\text { and discrimination) } \\
\text { Memory: } \\
\text { Short-term verbal } \\
\text { Numerical }\end{array}$ & $\begin{array}{l}\text { Performance post-inter- } \\
\text { vention was better on } \\
\text { mazes, pattern } \\
\text { match and same/ } \\
\text { different tasks }\end{array}$ & $\begin{array}{l}\text { Possible practice, learn- } \\
\text { ing and age con- } \\
\text { founds }\end{array}$ \\
\hline $\begin{array}{l}\text { Worobey \& } \\
\text { Worobey } \\
(1999) \\
\text { Study } 2^{(24)}\end{array}$ & 12 & $\begin{array}{l}\text { Sixteen children in two } \\
\text { groups: } \\
\text { (1) SBP, six female and } \\
\text { three male, age } \\
\text { range } 3 \text { years } 11 \\
\text { months to } 4 \text { years } 6 \\
\text { months } \\
\text { (2) Control, four female } \\
\text { and three male, age } \\
\text { range } 3 \text { years } 10 \\
\text { months to } 4 \text { years } 5 \\
\text { months }\end{array}$ & USA & $\begin{array}{l}\text { As Study } 1 \text { except: } \\
\text { School BF lower in } \\
\text { energy (about } \\
670 \mathrm{~kJ} \text { ) than usual BF } \\
\text { at home ( } 858 \mathrm{~kJ} \text { ) but } \\
\text { higher in } \mathrm{CHO} \text { and } \\
\text { lower in sugar }\end{array}$ & $\begin{array}{l}\text { Animal House (psycho- } \\
\text { motor) } \\
\text { MFFT } \\
\text { Cookie Hunt (visual per- } \\
\text { ception and classifi- } \\
\text { cation) } \\
\text { Same or different }\end{array}$ & $\begin{array}{l}\text { Better performance } \\
\text { after school BF than } \\
\text { controls } \\
\text { School BF showed } \\
\text { improvement on } \\
\text { Animal House, MFFT } \\
\text { and Same or different } \\
\text { tasks. More errors on } \\
\text { MFFT and Cookie } \\
\text { Hunt by controls than } \\
\text { school BF group }\end{array}$ & $\begin{array}{l}\text { Paired and independent } \\
t \text { tests performed. } \\
\text { Sample size too } \\
\text { small for ANOVA }\end{array}$ \\
\hline
\end{tabular}

QA, quality assessment; NCHS, National Center for Health Statistics; IG, independent groups; BF, breakfast; CT, cognitive test(s); SES, socio-economic status; IQ, intelligence quotient; DoH, Department of Health; RT, reaction time; np, quality assessment not possible; CHO, carbohydrate; MFFT, Matched Familiar Figures Test. 


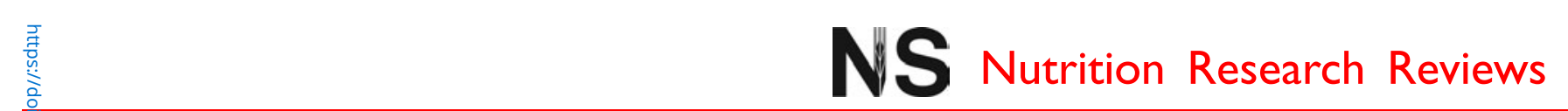

Table 5. Habitual breakfast in well-nourished children (four studies)

\begin{tabular}{|c|c|c|c|c|c|c|c|}
\hline Reference & QA & Sample & Country & Design & Cognitive measure & $\begin{array}{l}\text { Reported } \\
\text { findings }\end{array}$ & Comment \\
\hline $\begin{array}{l}\text { Herrero Lozano } \\
\text { et al. } \\
\quad(2006)^{(47)}\end{array}$ & 7 & $\begin{array}{l}141 \text { children (seventy-one } \\
\text { female, seventy male) } \\
\text { aged } 12-13 \text { years }\end{array}$ & Spain & $\begin{array}{l}\text { Four IG based on BF recalled } \\
\text { on previous day: } \\
\text { (a) Good quality (at least one } \\
\text { dairy, cereal, fruit) } \\
\text { (b) Improvable quality (lacks } \\
\text { one food group) } \\
\text { (c) Insufficient quality (lacks } \\
\text { two food groups) } \\
\text { (d) No BF } \\
\text { Quality of snack consumption } \\
\text { considered. Teachers blind } \\
\text { to condition }\end{array}$ & Scholastic performance & $\begin{array}{l}\text { Higher end-of-year school } \\
\text { marks associated with } \\
\text { increased BF quality. } \\
\text { Adding a good-quality } \\
\text { snack to poor-quality or } \\
\text { no BF increased per- } \\
\text { formance }\end{array}$ & - \\
\hline Lien $(2007)^{(82)}$ & 14 & $\begin{array}{l}7305 \text { children ( } 3694 \\
\text { female, } 3611 \text { male), age } \\
\text { range } 15-16 \text { years, } \\
74 \% \text { Norwegian/Wes- } \\
\text { tern }\end{array}$ & Norway & $\begin{array}{l}\text { Population-based survey of } \\
\text { parental education, family } \\
\text { structure, soft-drink intake, } \\
\text { smoking and dieting beha- } \\
\text { viour completed in junior } \\
\text { high school. BF frequency } \\
\text { (not quality) reported }\end{array}$ & $\begin{array}{l}\text { Average most recent grade for } \\
\text { mathematics, written Nor- } \\
\text { wegian, English and social } \\
\text { science on 0- to 6-point } \\
\text { scale. Dichotomised to } \leq 3 \\
\text { and }>3\end{array}$ & $\begin{array}{l}\text { Skipping BF ( } 0 \text { times per } \\
\text { week) associated with } \\
\text { twice likelihood of } \\
\text { grades } \leq 3 \text { in both } \\
\text { males and females. } \\
\text { Infrequent BF intake } \\
\text { (one or two times per } \\
\text { week) associated with } \\
\text { grades } \leq 3 \text { in males } \\
\text { only }\end{array}$ & $\begin{array}{l}\text { Adjustment for parental } \\
\text { education, family struc- } \\
\text { ture, soft-drink intake, } \\
\text { smoking and dieting } \\
\text { Part of Oslo Health Study } \\
2000-2001\end{array}$ \\
\hline $\begin{array}{l}\text { Lopez-Sobaler etal. } \\
\qquad(2003)^{(83)}\end{array}$ & 11 & $\begin{array}{c}180 \text { children, age range 9- } \\
13 \text { years; } 103 \text { male } \\
\text { (aged } 11.6 \text { (SD 1) years), } \\
\text { seventy-seven female } \\
\text { (aged } 11.4 \text { (SD 1) years) }\end{array}$ & Spain & $\begin{array}{l}\text { IG based on } 7 \mathrm{~d} \text { food diary } \\
\text { (a) Adequate BF: }>20 \% \text { daily } \\
\text { energy requirements } \\
\text { (b) Inadequate BF: }<20 \% \text { daily } \\
\text { energy requirements }\end{array}$ & $\begin{array}{l}\text { Scholastic aptitude test (verbal, } \\
\text { reasoning, calculation abilities) }\end{array}$ & $\begin{array}{l}\text { Better breakfast quality } \\
\text { associated with better } \\
\text { performance. Of chil- } \\
\text { dren with low scores, } \\
66 \% \text { had inadequate BF }\end{array}$ & $\begin{array}{l}\text { Accuracy issues related to } \\
\text { diary records. Statistical } \\
\text { analysis not powerful } \\
\text { Danone, España }\end{array}$ \\
\hline $\begin{array}{l}\text { Morales et al. } \\
\qquad(2008)^{(84)}\end{array}$ & 10 & $\begin{array}{l}467 \text { children aged } \\
12-17 \text { years }\end{array}$ & Spain & $\begin{array}{l}7 \mathrm{~d} \text { BF diary. Quality of BF } \\
\text { groups: } \\
\text { (a) Optimal: } 25 \% \text { daily energy } \\
\text { requirements, dairy, cer- } \\
\text { eals, fruits, fats } \\
\text { (b) Good: dairy, cereals, fruits, } \\
\text { fats } \\
\text { (c) Adequate: one food group } \\
\text { missing } \\
\text { (d) Inadequate: two food } \\
\text { groups missing } \\
\text { (e) No BF }\end{array}$ & $\begin{array}{l}\text { Scholastic performance (mean } \\
\text { score between } 2002-3 \text { for } \\
\text { range of subjects) }\end{array}$ & $\begin{array}{l}\text { Better BF quality associ- } \\
\text { ated with better mean } \\
\text { score }\end{array}$ & - \\
\hline
\end{tabular}

QA, quality assessment; IG, independent groups; BF, breakfast. 
cognitive test battery at $+180 \mathrm{~min}$ and found effects in children with lower IQ only. Benton et al. ${ }^{(41)}$ tested children between 140 and $210 \mathrm{~min}$ post-ingestion, with the possibility that the period between ingestion and testing varied between conditions and children.

There is less robust evidence for effects across other cognitive domains or between breakfasts providing similar energy but varying in macronutrient composition, GL or GI. It is difficult to confirm effects of breakfast quality in wellnourished children in mainstream education based on these acute studies.

\section{Studies of acute effects of breakfast in children of differing nutritional status}

There are seven studies that examined the effects of providing breakfast to children of differing nutritional status (see Table 3). Four studies were performed in South America, two in Jamaica and one in India. These studies compared the effects of breakfast in well-nourished and stunted and/or wasted children or children considered nutritionally at risk. Nutritional status was classified on the basis of height for age ( $-1 \mathrm{SD})$ and weight for age $(-0.5$ $\mathrm{SD}$ ) in four studies. These studies showed that cognitive performance was better following breakfast in the at-risk or undernourished group, with few if any effects on the wellnourished and not-at-risk control children. López et al. ${ }^{(42)}$ reported more errors in stunted children irrespective of treatment. However, some authors (for example, GranthamMcGregor $^{(15)}$ ) do not support the view that nutritional status is an important determinant of the effect of breakfast on cognitive function. In studies where details were provided, breakfasts delivered a substantial proportion of the energy requirements for these children.

Cognitive tasks susceptible to nutritional intervention in nutritionally vulnerable children appear to be verbal fluency and memory tasks, particularly short-term recognition (a hippocampal task), Sternberg memory search, as well as the matching familiar figures test (a measure of visual perception). It would appear that nutritionally at-risk children are more vulnerable in terms of memory performance and that these effects are not evident in geographically matched well-nourished control children.

\section{Studies of long-term effects of school breakfast programmes and breakfast clubs}

There are thirteen studies that examined breakfast provision at school (see Table 4). Generally, these evaluated government-funded breakfast provision which was free to low-income children. Of the studies, seven were conducted in the USA in children of low socio-economic status, one study took place in the UK and the rest were undertaken in South America (in undernourished, at-risk children), South Africa and Jamaica. The interventions employed were predominantly school breakfast $v$. no school breakfast (either breakfast at home or no breakfast). The duration of the school breakfast programme during these studies ranged from 4 weeks to 3 years but the majority of evaluation studies had a duration of 6-12 weeks. The children in these studies tended to be younger (between 3 and 8 years of age) than in other studies included in the present review. No studies considered effects in adolescents.

Scholastic achievement tests were used as measures of cognitive function in seven studies. These studies, taken together, showed improvement mainly in mathematics or arithmetic scores post-intervention. School breakfast programmes were associated with increased attendance or decreased absenteeism, a possible explanation for the improved performance. This is especially likely where scholastic achievement tests are employed as postintervention measures. Benefits were not greater or confined to undernourished or at-risk groups in studies that also included well-nourished controls with one exception ${ }^{(43)}$.

In seven studies specific tests of cognitive function were employed. Of these, one study ${ }^{(44)}$ showed improved memory, another reported improved concentration ${ }^{(45)}$ while a third found no effect on a range of tests ${ }^{(23)}$. Worobey \& Worobey ${ }^{(24)}$ found a positive effect of a school breakfast programme on a range of mainly spatial cognitive tests in two separate samples of school children. Cueto \& Chinen $^{(46)}$ report acute effects of breakfast provision in fullgrade and multiple-grade schools, confined to tests of memory, arithmetic and, to a lesser degree, reading in the multiple-grade schools. Multiple-grade schools include children of different ages within the same class. They are associated with more poverty and lower achievement than full-grade schools and are therefore more likely to include children who are nutritionally at risk.

Provision of breakfast at school seems to have positive effects in all but two studies, particularly in these younger children who were participating in a free, universal school feeding programme. Despite the lack of detail of the nutrient composition and energy provided by the breakfasts at school, the effects of breakfast seem positive. These effects could, however, be an artifact of the increased school attendance that such provision encourages. One pitfall of school breakfast programmes is the potentially negative impact of breakfast provision on class time and pupil-teacher contact. This depends on whether breakfast is provided before the school day or during time normally allocated for teaching. In some studies, particularly in the developing world, breakfast was provided during teaching time ${ }^{(46)}$.

\section{Studies of effects of habitual breakfast quality}

There were only four studies that considered habitual breakfast intake (see Table 5). Of the studies, three were conducted in Spain, utilising food diaries to determine the quality of breakfast intake based on target food groups. These studies suggest a positive effect of breakfast quality on scholastic performance. It was indicated by one study that snack provision could ameliorate the negative effect of a poor-quality or no breakfast ${ }^{(47)}$. The studies did not include measures of cognitive function other than school performance.

\section{Discussion}

The present review has identified relatively few goodquality studies that examine the effects of breakfast on the cognitive performance of school-aged children. We 
identified forty-five studies presented in forty-two articles published between 1950 and 2008 as suitable for inclusion, although many lacked scientific rigour. This is despite intense public and scientific interest and confident claims in the media on behalf of governments and the nutrition industry regarding the effects of breakfast. The majority of the studies reviewed were sponsored in whole or in part by industry. A recent development is the consideration of the impact of habitual breakfast on performance. The four studies in this category were all conducted in the last decade. Across all categories there is a predominance of studies in younger children and far fewer in adolescents in whom metabolic and cognitive effects could be different.

Overall, the quality of studies was poor. Some studies were not counterbalanced or allocation to condition was not randomised. It was sometimes unclear whether testing was performed blind to treatment condition where possible. Socio-economic status, if specified and not deliberately selected for, was predominantly middle class and monetary incentives were provided to parents in a number of studies. Studies included in Table 2 assume that the children were well nourished since they were all described as healthy and no consideration of the nutritional or weight status of the sample was provided. However, it is probable that samples included children across a range of body weights but these were not reported. Weight is likely to be positively skewed, reflecting the distribution of body weight in the populations from which they were recruited (i.e. predominantly white, middle class). Quality assessment scores reflected almost the whole range of possible scores (range 4-17). Differences in quality scores by category were small and decade of publication did not appear to influence study quality. However, habitual breakfast studies tended to score lower than other categories probably due to confounds inherent in these designs. Lifestyle factors are difficult to account for, socio-economic status may be associated with breakfast quality, and the free-living nature of the studies and reliance on food diaries reduce experimental control.

Arguably, studies in undernourished children are more difficult to design and execute. However, the studies of undernourished or at-risk children reviewed were equally well performed, scoring across a similar range to the acute healthy investigations (9-16 out of a maximum 18). The majority employed repeated-measures designs (five studies), details of breakfasts provided were good, the cognitive tests employed comparable and, where stated, the analysis was appropriate.

Difficulties also present in evaluations of school breakfast programmes. These are logistically challenging to conduct since it is difficult to match samples and the introduction of contamination between treatment arms can have a serious impact on the study. For example, Shemilt et al. ${ }^{(45)}$ experienced high attrition and contamination, such that participants in the intervention and non-intervention arms became aware of each other's condition. Therefore, the data could not be analysed using the intended method.

\section{Methodological issues}

Breakfast intervention. Breakfast manipulations were often not matched for energy or volume and no mention is made of palatability, which could affect cognitive, affective and behavioural responses. Studies varied in the amount of each breakfast consumed, rendering conditions unmatched in terms of energy content when the variable of interest was carbohydrate quality or GL. In this respect studies were generally poorly conducted. Few firm conclusions can be made from studies of school breakfast programmes which do not tend to record or report the nature or quantity of the breakfast. Habitual breakfast studies rely on food diaries which are subject to the same bias of under- and overreporting in children as in adults ${ }^{(48)}$.

Often, choice of breakfast intervention was not driven by a priori hypotheses about the mechanisms by which breakfast could have an impact on cognitive performance. School breakfast programme evaluations are bound by the constraints of the programme. Other studies may be guided by the commercial interests of the study sponsor. However, recent studies have attempted to evaluate whether food characteristics such as GI and GL are related to the effects of breakfast on cognitive function ${ }^{(39,41)}$. Ingwersen et al. ${ }^{(39)}$ found advantageous performance after a low-GI breakfast whereas Benton et al. ${ }^{(41)}$ report benefits after low-GL breakfasts. Moreover, Benton et al. ${ }^{(41)}$ calculated the GL of the breakfasts used in Ingwersen et al.' ${ }^{(39)}$ study and report that the low-GI breakfast was also low GL. Hence it is difficult to attribute the effects observed to GI or GL. There is also no agreement as to the relative importance of GI $v$. GL in terms of which best predicts health outcomes in adults ${ }^{(49)}$. Importantly, these studies have not profiled the metabolic response to breakfasts varying in GI or GL in children and the assumption is that the responses are the same as in adults.

Cognitive performance testing. Some issues relate to the appropriateness of cognitive testing employed by the studies to date in terms of their difficulty level, style and cognitive domain under study. Moreover, the suitability of some of the tests employed for children of different ages, developmental stages and intellectual level is often not considered. The cognitive tests employed in experimental studies were fairly limited, and these were not necessarily selected for their sensitivity to nutrient intervention or change over time. Some studies used global neuropsychological tests, more usually employed for diagnostic purposes. Across studies, tests were not readily comparable and accuracy and error rates were not provided by all studies. Although ecologically valid, end-of-year school performance may not provide the most sensitive indicator of the effect of a school breakfast programme. Many studies do not control for other factors which are likely to influence school grade, including home environment, parental involvement, school system and quality.

Performance on many of the cognitive tasks is evaluated in terms of accuracy scores. Little consideration is given to motivation and effort including the ability to sustain performance over time. However, breakfast consumption might facilitate motivation and reduce the underlying 'maintenance costs' of sustained performance. Sustaining concentration and retaining information are cognitive processes of key importance for scholastic achievement. The cost of maintaining these processes may vary between 
children and this may be a partial explanation why positive effects of breakfast are most easily identifiable in nutritionally at-risk children. Thus, future assessment of cognitive performance should include measures of motivation, such as number of trials attempted or frustration tolerance, in addition to accuracy.

Cognitive performance testing: design. Studies in adults suggest that glucoregulation following macronutrient manipulation, rather than absolute levels of blood glucose, may be most important for cognition ${ }^{(50,51)}$. Better glucoregulation has been associated with superior shortterm and delayed verbal memory ${ }^{(52)}$. Smaller blood glucose excursions, rather than sharp fluctuations, reflect lighter metabolic stress and may be better for cognition. If glucoregulatory processes moderate the relationship between food and cognition in children, then repeatedmeasures designs are necessary. Participants are likely to demonstrate inter-individual variability in glucoregulation, and independent-groups designs will not control for the potential error that this introduces. This issue is of particular importance in studies of children because of their relatively faster metabolism ${ }^{(13)}$.

Cognitive performance testing: analysis. In some of the studies reviewed, the statistical analysis was inappropriate to the design of the study. Often more powerful analyses could have been performed. Some studies failed to describe the statistical procedures and no or limited critical values were reported, precluding meta-analyses of these data. The age of the study had some bearing on the complexity of the statistical analysis performed, with older studies tending towards simpler, non-parametric statistical procedures, possibly due to the lack of available computing power.

Mechanisms. Mechanisms for the facilitation of cognitive performance by breakfast are not well established ${ }^{(8)}$. There are likely to be different mechanisms of action responsible for short- and long-term effects of breakfast consumption. Moreover, these purported mechanisms may not necessarily be the same in adults as in young children, whose brain metabolic requirements are relatively much greater than those of the adult brain. In addition, metabolic processes of adolescents' brains begin to resemble those of adults during puberty but do not reach adult levels until $16-18$ years $^{(13)}$.

Mechanisms: physiological. While some studies suggest that glucose ingestion facilitates cognitive performance ${ }^{(53-56)}$, others report no direct relationship between performance and glucose levels following breakfast consumption $^{(57,58)}$. Rather than a direct effect of glucose on cognitive performance, the mechanism of action may involve one or many correlates of blood glucose. Glucose ingestion gives rise to changes in levels of acetylcholine, insulin, serotonin, glutamate and cortisol, all of which can affect cognitive function ${ }^{(58-61)}$. It is possible that any or a combination of these central and peripheral changes are involved in the impact of breakfast on performance.

Therefore, we are currently lacking a strong theoretical or evidence base to relate specific neurochemical or physiological activity to specific cognitive functions. These potential biomarkers for cognitive function are more difficult to measure than the actual function itself. This is particularly pertinent in children in whom invasive measures are ethically and practically difficult. Some physiological measures of autonomic nervous system activity such as heart rate and electroencephalogram which have been shown to vary in relation to cognitive demand ${ }^{(62)}$ may be more easily applied in studies of children. Appropriate methods to track biomarkers during cognitive activity are required in order to elucidate the mechanisms by which breakfast may affect performance.

The use of physiological biomarkers relies on the assumption that peripheral measures reflect central activity. Indeed, peripheral glucose and its metabolites are well regulated in healthy individuals to maintain homeostasis and elicit appropriately rapid postprandial responses. Peripheral glucose regulation and metabolism in children may not be the same as in adults ${ }^{(63)}$.

In the long term, breakfast consumption may lead to beneficial physiological changes in nutrient status. Thus positive effects of breakfast on cognitive performance may be the product of better nutritional profiles rather than transient changes in blood parameters. Achieving better nutrient and vitamin status or rectifying deficiencies, as demonstrated by some studies in the developing world, may be responsible for the effects seen on performance.

\section{Behavioural mechanisms}

While physiological explanations for the effects of breakfast on cognitive performance are appealing, behavioural mechanisms may play an important role in the cognitive response to breakfast. In the short term, breakfast consumption may function to heighten subjective feelings of alertness and motivation to concentrate and learn. This could occur because of the learned association between breakfast consumption and feelings of wellbeing, or the reduction of hunger (for example, Wesnes et al. ${ }^{(33)}$, Dye \& Blundell $^{(9)}$ and Gibson ${ }^{(58)}$ ). Unfortunately, few studies incorporate measures of both appetite and mood alongside objective cognitive measures.

In the long term, studies indicate that school breakfast programmes increase scholastic performance. This outcome could be a direct effect of the repeated consumption of breakfast and the development of a learned association of this with wellbeing or ability to concentrate. Alternatively, positive effects could be a result of the improved nutritional state which is known to result from regular breakfast consumption $^{(1,64)}$. The effects seen in response to school breakfast programmes could simply be explained as an artifact of increased school attendance, motivated to attend by the provision of the breakfast ${ }^{(23,29,46)}$.

\section{Conclusions}

Overall, evidence suggests that breakfast consumption has generally positive effects on cognitive performance in comparison with breakfast omission. This effect appears to be pervasive in both acute studies and longer-term school breakfast programmes. However, the apparent beneficial effects of school breakfast programmes may be linked to 
increased attendance and reduced absenteeism, and effects of such provision in older children are not known. In addition, breakfast effects are more easily demonstrable in nutritionally vulnerable children.

It is difficult to recommend an optimal breakfast for cognitive function based on the currently available research. One study has suggested that solid breakfasts may be advantageous over liquid breakfasts ${ }^{(33)}$, perhaps due to differing rates of gastric emptying. In addition, some studies indicate that low-GI or low-GL breakfasts may confer benefits but it is hard to differentiate between these two indicators of glycaemic response in the few studies that have examined these food characteristics.

The majority of studies have concentrated on the measurement of memory and attention performance, with less examination of tasks that engage other cognitive domains, processes and aptitudes. There have been few examinations of problem solving and psychomotor skill, for example. However, from the studies reviewed, it is difficult to conclude which specific cognitive domains are most sensitive to nutritional manipulations at breakfast time, although there is most abundant support for effects on memory.

\section{Recommendations for future work}

The present review has highlighted problems in this research area which could be addressed by the following recommendations for future work.

Future studies should test focused hypotheses, based on a small number of theoretically selected breakfast conditions, presented according to carefully counterbalanced repeatedmeasures designs with large samples which yield sufficient power. Breakfasts must be matched for energy but should differ sufficiently in key features, for example, macronutrient composition, style or glycaemic response, in order to experimentally investigate potential mechanisms of action. Tasks that span a wide range of cognitive domains with demonstrated sensitivity to nutritional manipulations should be used so that null findings can be ascribed to true lack of effect rather than test insensitivity. In acute studies, effects of breakfast may be more pronounced some time after consumption when the metabolic challenge of food consumption has subsided. Late-morning testing rather than immediate post-breakfast testing merits further exploration. Enduring and meaningful effects are likely to result from chronic interventions of at least 12 weeks with appropriate timing of the tests of cognitive and/or scholastic performance. Lastly, the present review has highlighted the need for greater examination of the effect of breakfast in adolescents.

\section{Acknowledgements}

All authors contributed equally to the preparation of the paper.

A. H. was supported by an Economic and Social Research Council (ESRC) research studentship.

L. D. was supported by a grant from Kellogg Company UK to prepare the present review.

A. H. and C. L. L. have no conflicts of interest.

\section{References}

1. Ruxton CHS \& Kirk TR (1997) Breakfast: a review of associations with measures of dietary intake, physiology and biochemistry. Br J Nutr 78, 199-213.

2. de la Hunty A \& Ashwell M (2006) Are people who regularly eat breakfast cereals slimmer than those who don't? A systematic review of the evidence. Nutr Bull 32, 118-128.

3. Hansen K \& Joshi H (2008) Millennium Cohort Study Third Survey: A User's Guide to Initial Findings, p. 162. London: Centre for Longitudinal Studies, Institute of Education.

4. Sjöberg A, Hallberg L, Höglund D, et al. (2003) Meal pattern, food choice, nutrient intake and lifestyle factors in The Göteborg Adolescence Study. Eur J Clin Nutr 57, 1569-1578.

5. Haines PS, Guilkey DK \& Popkin BM (1996) Trends in breakfast consumption of US adults between 1965-1991. J Am Diet Assoc 96, 464-470.

6. Street C \& Kenway P (1999) Food for thought: breakfast clubs and their challenges. London: New Policy Institute.

7. Dye L, Lluch A \& Blundell JE (2000) Macronutrients and mental performance. Nutrition 16, 1021-1034.

8. Hoyland A, Lawton CL \& Dye L (2008) Acute effects of macronutrient manipulations on cognitive test performance in healthy young adults: a systematic research review. Neurosci Biobehav Rev 32, 72-85.

9. Dye L \& Blundell JE (2002) Functional foods: psychological and behavioural functions. Br J Nutr 88, Suppl. 2, 187-211.

10. Sokoloff L (1976) Circulation and energy metabolism. In Basic Neurochemistry, pp. 388-413 [GJ Siegel, RW Albers and BW Agranoff, editors]. Boston: Little Brown.

11. Kennedy D \& Sokoloff L (1957) An adaptation of the nitrous oxide method to the study of the cerebral circulation in children; normal values for cerebral blood flow and cerebral metabolic rate in childhood. J Clin Invest 36, 1130-1137.

12. Chugani HT (1994) Development: of regional brain glucose metabolism in relation to behaviour and plasticity. In Human Behavior and the Developing Brain, pp. 153-175 [G Dawson and KW Fischer, editors]. New York: Guildford Press.

13. Chugani HT (1998) A critical period of brain development: studies of cerebral glucose utilization with PET. Prev Med 27, 184-188.

14. Connors CK \& Blouin AG (1983) Nutritional effects on behaviour of children. J Psychiatr Res 17, 198-201.

15. Grantham-McGregor S (2005) Can the provision of breakfast benefit school performance? Food Nutr Bull 26, Suppl. 2, $144-158$.

16. Taras H (2005) Nutrition and student performance at school. J Sch Health 75, 199-213.

17. Pollitt E \& Mathews R (1998) Breakfast and cognition: an integrative summary. Am J Clin Nutr 67, Suppl., 804-813.

18. Ells LJ, Hillier FC, Shucksmith J, et al. (2008) A systematic review of the effect of dietary exposure that could be achieved through normal dietary intake on learning and performance of school-aged children of relevance to UK schools. Br J Nutr 100, 927-936.

19. Kristjansson EA, Robinson V \& Petticrew M, et al. (2007) School feeding for improving the physical and psychosocial health of disadvantaged elementary school children. The Cochrane Database of Systematic Reviews 2007, issue 1, CD004676. http://www.mrw.interscience.wiley.com/ cochrane/clsysrev/articles/CD004676/frame.html

20. Lichtenstein AH, Yetley EA \& Lau J (2008) Application of systematic review methodology to the field of nutrition. J Nutr 138, 2297-2306.

21. Dickie NH \& Bender AE (1982) Breakfast and performance in schoolchildren. Br J Nutr 48, 483-496. 
22. Mahoney CR, Taylor HA, Kanarek RB, et al. (2005) Effect of breakfast composition on cognitive processes in elementary school children. Physiol Behav 85, 635-645.

23. Pollitt E, Jacoby E \& Cueto S (1996) School breakfast and cognition among nutritionally at-risk children on the Peruvian Andes. Nutr Rev 54, 22-26.

24. Worobey J \& Worobey HS (1999) The impact of a two-year school breakfast program for preschool-aged children on their nutrient intake and pre-academic performance. Child Stud J 29, 113-131.

25. Chandler AM, Walker SP, Connolly K, et al. (1995) School breakfast improves verbal fluency in undernourished Jamaican children. J Nutr 125, 894-900.

26. Pollitt E, Cueto S \& Jacoby ER (1998) Fasting and cognition in well- and undernourished schoolchildren: a review of three experimental studies. Am J Clin Nutr 67, Suppl., 779-784.

27. Simeon DT \& Grantham-McGregor S (1989) Effects of missing breakfast on cognitive functions of schoolchildren of differing nutritional status. Am J Clin Nutr 49, 646-653.

28. Simeon D \& Grantham-McGregor S (1987) Cognitive function, undernutrition and missed breakfast. Lancet ii, 737-738.

29. Simeon DT (1998) School feeding in Jamaica: a review of its evaluation. Am J Clin Nutr 67, Suppl., 790-794.

30. Jadad AR, Moore RA, Carroll D, et al. (1996) Assessing the quality of reports of randomised clinical trials: is blinding necessary? Control Clin Trials 17, 1-12.

31. Thomas J, Sutcliffe K, Harden A, et al. (2003) Children and Healthy Eating: A Systematic Review of Barriers and Facilitators. London: EPPI Centre, Social Science Research Unit, Institute of Education, University of London.

32. Jackson C, Cheater F \& Reid I (2008) A systematic review of decision support needs of parents making child health decisions. Health Expect 11, 232-251.

33. Wesnes KA, Pincock C, Richardson D, et al. (2003) Breakfast reduced declines in attention and memory over the morning in schoolchildren. Appetite 41, 329-331.

34. Busch CR, Taylor HA, Kanarek RB, et al. (2002) The effects of a confectionery snack on attention in young boys. Physiol Behav 77, 333-340.

35. Pollitt E, Leibel RL \& Greenfield D (1981) Brief fasting, stress and cognition in children. Am J Clin Nutr 34, 1526-1533.

36. Vaisman N, Voet H, Akivis A, et al. (1996) Effect of breakfast timing on the cognitive functions of elementary school students. Arch Pediatr Adolesc Med 150, 1089-1092.

37. Cromer BA, Tarnowski KJ, Stein AM, et al. (1990) The school breakfast program and cognition in adolescents. $J$ Dev Beh Pediatr 11, 295-300.

38. Pollitt E, Lewis NL, Garza C, et al. (1982-3) Fasting and cognitive function. J Psychiatr Res 17, 169-174.

39. Ingwersen J, Defeyter MA, Kennedy DO, et al. (2007) A low glycaemic index breakfast cereal preferentially prevents children's cognitive performance from declining throughout the morning. Appetite 49, 240-244.

40. Atkinson FS, Foster-Powell K \& Brand-Miller JC (2008) International tables of glycemic index and glycemic load values: 2008. Diabetes Care 31, 2281-2283.

41. Benton D, Maconie A \& Williams C (2007) The influence of the glycaemic load of breakfast on the behaviour of children in school. Physiol Behav 92, 717-724.

42. López I, de Andraca I, Perales CG, et al. (1993) Breakfast omission and cognitive performance of normal, wasted and stunted schoolchildren. Eur J Clin Nutr 47, 533-542.

43. Richter LM, Rose C \& Griesel RD (1997) Cognitive and behavioural effects of a school breakfast. S Afr Med J $\mathbf{8 7}$, Suppl. 1, 93-100.
44. Vera Noriega JA, Dominguez Ibanez SE, Pena Ramos MO, et al. (2000) Evaluation of the effects of a school breakfast program on attention and memory. Arch Latinoam Nutr 50, $35-41$.

45. Shemilt I, Harvey I, Shepstone L, et al. (2004) A national evaluation of school breakfast clubs: evidence from a cluster randomized controlled trial and an observational analysis. Child Care Health Dev 30, 413-427.

46. Cueto S \& Chinen M (2008) Educational impact of a school breakfast programme in rural Peru. Int $J$ Educ Dev 28, $132-148$.

47. Herrero Lozano R \& Fillat Ballesteros JCF (2006) A study on breakfast and school performance in a group of adolescents. Nutr Hosp 21, 346-352.

48. Baxter SD, Hardin JW, Royer JA, et al. (2008) Children's recalls from five dietary-reporting validation studies. Intrusions in correctly reported and misreported options in school breakfast reports. Appetite 51, 489-500.

49. Livesey G, Taylor R, Hulshof T, et al. (2008) Glycemic response and health - a systematic review and meta-analysis: the database, study characteristics, and macronutrient intakes. Am J Clin Nutr 87, Suppl. 1, 223-236.

50. Meikle A, Riby LM \& Stollery B (2004) The impact of glucose ingestion and gluco-regulatory control on cognitive performance: a comparison of younger and middle aged adults. Hum Psychopharmacol 19, 523-535.

51. Messier C, Gagnon M \& Knott V (1997) Effect of glucose and peripheral glucose regulation on memory in the elderly. Neurobiol Aging 18, 297-304.

52. Nabb S \& Benton D (2006) The influence on cognition of the interaction between the macro-nutrient content of breakfast and glucose tolerance. Physiol Behav 87, 16-23.

53. Benton D, Owens DS \& Parker PY (1994) Blood glucose influences memory and attention in young adults. Neuropsychologia 32, 595-607.

54. Foster J, Lidder P \& Sunram S (1998) Glucose and memory: fractionation of enhancement effects? Psychopharmacology 137, 259-270.

55. Kennedy D \& Scholey A (2000) Glucose administration, heart rate and cognitive performance: effects of increasing mental effort. Psychopharmacology 149, 63-71.

56. Sünram-Lea S, Foster J, Durlach P, et al. (2002) The effect of retrograde and anterograde glucose administration on memory performance in healthy young adults. Behav Brain Res 134, 505-516.

57. Bellisle F (2001) Glucose and mental performance. Br J Nutr 86, 117-118.

58. Gibson EL (2007) Carbohydrates and mental function: feeding or impeding the brain? Nutr Bull 32, Suppl. 1, 71-83.

59. Hasselmo ME \& Giocomo LM (2006) Cholinergic modulation of cortical function. J Mol Neurosci 30, 133-135.

60. Park CR (2001) Cognitive effects of insulin in the central nervous system. Neurosci Biobehav Rev 25, 311-323.

61. Schmitt JAJ, Jorissen BL, Dye L, et al. (2005) Memory function in women with premenstrual complaints and the effect of serotonergic stimulation by acute administration of an $\alpha$-lactalbumin protein. $J$ Psychopharmacol 19, 375-384.

62. Fairclough SH \& Houston K (2004) A metabolic measure of mental effort. Biol Psychol 66, 177-190.

63. Beardsall K, Yuen K, Williams R, et al. (2006) Applied physiology of glucose control. Curr Paediatr 16, 434-438.

64. Rampersaud GC, Pereira MA, Girard BL, et al. (2005) Breakfast habits, nutritional status, body weight, and academic performance in children and adolescents. $J$ Am Diet Assoc 105, 743-760. 
65. Ma G, Hu X, Gao S, et al. (1999) Effect of energy intake at breakfast on school performance. Wei Sheng Yan Jiu $\mathbf{2 8}$, 286-288.

66. Marquez Acosta M, Sutil de Naranjo R, Rivas de Yepez CE, et al. (2001) Influence of breakfast on cognitive functions of children from an urban area in Valencia, Venezuela. Arch Latinoam Nutr 51, 57-63.

67. Michaud C, Musse N, Nicolas JP, et al. (1991) Effects of breakfast-size on short-term memory, concentration, mood and blood glucose. J Adolesc Health 12, 53-57.

68. Morrell G \& Atkinson DR (1977) Effects of breakfast program on school performance and attendance of elementary school children. Education 98, 111-116.

69. Morris N \& Sarll P (2001) Drinking glucose improves listening span in students who miss breakfast. Educ Res 43, 201-207.

70. Widenhorn-Müller K, Hille K, Klenk J, et al. (2008) Influence of having breakfast on cognitive performance and mood in 13- to 20-year-old high school students: results of a crossover trial. Pediatrics 122, 279-284.

71. Wyon DP, Abrahamsson L, Jartelius M, et al. (1997) An experimental study on the effects of energy intake at breakfast on the test performance of 10-year-old children in school. Int J Food Sci Nutr 48, 5-12.

72. Cueto S, Jacoby E \& Pollitt E (1998) Breakfast prevents delays of attention and memory functions among nutritionally at-risk boys. J Appl Dev Psychol 19, 219-234.

73. Grantham-McGregor SM, Chang S \& Walker SP (1998) Evaluation of school feeding programs: some Jamaican examples. Am J Clin Nutr 67, Suppl., 785-789.

74. Chandler AK, Walker SP, Connolly K, et al. (1995) School breakfast improves verbal fluency in undernourished Jamaican children. J Nutr 125, 894-900.
75. Muthayya S, Thomas T, Srinivasan K, et al. (2007) Consumption of a mid-morning snack improves memory but not attention in school children. Physiol Behav 90, $142-150$.

76. Noriega JAN (2000) Method and theory in school breakfast program evaluation. Estudios de Psicologia 5, 33-48.

77. Kleinman RE, Hall S, Green H, et al. (2002) Diet, breakfast and academic performance in children. Ann Nutr Metab 46, Suppl. 1, 24-30.

78. Lieberman HM, Hunt IF, Coulson AH, et al. (1976) Evaluation of a ghetto school breakfast program. J Am Diet Assoc 69, 132-138.

79. Meyers AF, Sampson AE, Weitzman M, et al. (1989) School Breakfast Program and school performance. Am J Dis Child 143, 1234-1239.

80. Murphy JM, Pagano M, Nachmani J, et al. (1998) The relationship of school breakfast to psychosocial and academic functioning: cross-sectional and longitudinal observations in an inner-city school sample. Arch Pediatr Adolesc Med 152, 899-907.

81. Wahlstrom KL \& Begalle M (1999) More than test scores results of the Universal School breakfast pilot in Minnesota. Topics Clin Nutr 15, 7-29.

82. Lien L (2007) Is breakfast consumption related to mental distress and academic performance in adolescents? Public Health Nutr 10, 422-428.

83. Lopez-Sobaler AM, Ortega RM, Quintas ME, et al. (2003) Relationship between habitual breakfast and intellectual performance (logical reasoning) in well-nourished schoolchildren of Madrid (Spain). Eur J Clin Nutr 57, Suppl. 1, 49-53.

84. Morales IF, Villas MVA, Vega CJM, et al. (2008) Relation between the breakfast quality and the academic performance in adolescents of Guadalajara (Castilla-La Mancha). Nutr Hosp 23, 383-387. 


\section{Appendix: Quality assessment tool}

\section{Quality assessment sheet: Breakfast and performance in children review}
A. Overview of study
$1,2,3,4,12,17,18$
B. Data collection
$5,6,7,8$
C. Manipulation
$9,10,11$
D. Outcomes and analysis
$13,14,15,16$

Paper:

Rater:

Score 0 if criterion not satisfied. Score 1 if criterion

satisfied.

Score:

Clear description of setting/environment, e.g. location, laboratory/classroom

Clear description of sample, e.g. age (mean, SD, range), sex, $n$

Clear description of study design

Clear description of data collection

Provision of recruitment data and strategy

Provision of attrition data

Provision of compliance data, i.e. performance testing and breakfast intake

Clear description of manipulation, e.g. serving size, composition, kJ

Appropriateness of manipulation, e.g. serving size, food style/quality

Evidence of fasting before testing

Sufficiency of matching, sample selection, blinding, counterbalancing or placebo comparison

N.B. within limitations of study design

Valid and reliable outcomes, e.g. appropriateness of cognitive test

Clear description of data analysis

Appropriateness of data analysis

Clear description of findings

Strengths of study and suggestions for future work

Limitations of study 